\title{
Stress Impairs GABAergic Network Function in the Hippocampus by Activating Nongenomic Glucocorticoid Receptors and Affecting the Integrity of the Parvalbumin-Expressing Neuronal Network
}

\author{
Wen Hu', Mingyue Zhang ${ }^{2,3,4}$, Boldizsár Czéh ${ }^{1,5}$, Gabriele Flügge ${ }^{1,3,6}$ and Weiqi Zhang*,2,3,4,6 \\ 'Clinical Neurobiology Laboratory, German Primate Center, Leibniz Institute for Primate Research, Göttingen, Germany; ${ }^{2}$ Department of \\ Neurophysiology, Center of Physiology and Pathophysiology, University of Göttingen, Göttingen, Germany; ${ }^{3}$ DFG Research Center Molecular \\ Physiology of the Brain, University of Göttingen, Göttingen, Germany; ${ }^{4}$ Laboratory of Molecular Psychiatry, Department of Psychiatry, Westfälische \\ Wilhelms University, Münster, Germany
}

\begin{abstract}
Stress facilitates the development of psychiatric disorders in vulnerable individuals. It affects physiological functions of hippocampal excitatory neurons, but little is known about the impact of stress on the GABAergic network. Here, we studied the effects of stress and a synthetic glucocorticoid on hippocampal GABAergic neurotransmission and network function focusing on two perisomatic interneurons, the parvalbumin (PV)- and the cholecystokinin (CCK)-positive neurons. In acute hippocampal slices of rat, application of the potent glucocorticoid receptor (GR) agonist dexamethasone (DEX) caused a rapid increase in spontaneous inhibitory postsynaptic currents (sIPSCS) in CAI pyramidal neurons. This effect was mediated by a nongenomic GR that evoked nitric oxide (NO) release from pyramidal neurons. Retrograde NO signaling caused the augmentation of GABA release from the interneurons and increased CCK release, which in turn further enhanced the activity of the PV-positive cells. Interestingly, chronic restraint stress also resulted in increased sIPSCs in CAI pyramidal neurons that were $\mathrm{Ca}^{2+}$-dependent and an additional DEX application elicited no further effect. Concomitantly, chronic stress reduced the number of PV-immunoreactive cells and impaired rhythmic sIPSCs originating from the PV-positive neurons. In contrast, the CCK-positive neurons remained unaffected. We therefore propose that, in addition to the immediate effect, the sustained activation of nongenomic GRs during chronic stress injures the PV neuron network and results in an imbalance in perisomatic inhibition mediated by the PV and CCK interneurons. This stress-induced dysfunctional inhibitory network may in turn impair rhythmic oscillations and thus lead to cognitive deficits that are common in stress-related psychiatric disorders.

Neuropsychopharmacology (2010) 35, 1693-1707; doi:10.1038/npp.2010.31; published online 31 March 2010
\end{abstract}

Keywords: GABA; parvalbumin; chronic stress; cholecystokinin; neuroplasticity; nitric oxide

\section{INTRODUCTION}

Severe or chronic stress can facilitate the development of psychiatric illnesses in genetically predisposed individuals. Preclinical studies document that chronic stress has a significant impact on neuroplasticity in limbic brain areas

*Correspondence: Professor Dr W Zhang, Laboratory of Molecular Psychiatry, Department of Psychiatry, University of Münster, AlbertSchweitzer-Str. II, 48|49 Münster, Germany, Tel: + 49-25I-83566I0, Fax: + 49-251-8357|28, E-mail: wzhang@uni-muenster.de or Professor Dr G Flügge, Clinical Neurobiology Laboratory, German Primate Center, Kellnerweg 4, 37077 Göttingen, Germany, Tel: + 49-55 I-385II33, Fax: + 49-55 I-385 I 307, E-mail: gluegg@gwdg.de

${ }^{5}$ Current address: Molecular Neurobiology, Max-Planck-Institute of Psychiatry, Munich, Germany.

${ }^{6}$ These authors contributed equally to this work.

Received 2 October 2009; revised 18 January 2010; accepted 18 February 2010 and that these changes have been suggested to contribute to the pathophysiology of mood disorders (Drevets et al, 2008; McEwen, 2007; Pittenger and Duman, 2008). Stress increases the levels of circulating corticosteroids whose actions are mediated by mineralocorticoid and glucocorticoid receptors (MRs and GRs), which are abundantly expressed in the brain and influence emotional and cognitive functions (Joëls et al, 2007; McEwen et al, 1986). As MRs and GRs are expressed predominantly in principal cells, previous studies concerning corticosteroid effects on neuronal activity in the hippocampus focused mainly on these neurons (Joëls et al, 2007; Joëls, 2008; Karst et al, 2005), whereas the GABAergic network remained relatively unexplored. However, microdialysis studies report on increased extracellular GABA levels in the hippocampus of rats exposed to a psychological stressor (de Groote and Linthorst, 2007). Stress or artificially elevated glucocorticoid levels, which have been shown to regulate the expression of 
glutamic acid decarboxylase (GAD) (Bowers et al, 1998; Stone et al, 2001), caused an increase in the magnitude of inhibitory postsynaptic currents (IPSCs) in the hippocampus (Maggio and Segal, 2009). Parallel to these findings, accumulating evidences from clinical studies suggest a dysregulation of the GABAergic system in depressed patients (Brambilla et al, 2003; Hasler et al, 2007; Krystal et al, 2002; Sanacora et al, 1999).

Diverse subtypes of GABAergic interneurons provide networks of inhibition that sculpt the firing pattern of pyramidal cells and orchestrate network oscillations that represent distinct brain states (Buzsáki and Draguhn, 2004; Somogyi and Klausberger, 2005). A specific subtype of GABAergic neurons that innervate the perisomatic domain of principal neurons has a pivotal role in generating synchronized network oscillations (Somogyi and Klausberger, 2005; Klausberger et al, 2005). Driven by external and internal stimuli, these neurons evoke rhythmic perisomatic hyperpolarizations in principal neurons to accurately control the timing of firing and to synchronize the activity of a large number of pyramidal cells (Somogyi and Klausberger, 2005; Klausberger et al, 2005; Sohal et al, 2009). Precise synchronous activity of oscillating networks is essential for mediating complex cognitive processes such as perception and memory (Buzsáki and Draguhn, 2004; Somogyi and Klausberger, 2005).

In this study, we focus on the two major perisomatic interneurons: parvalbumin (PV) and cholecystokinin (CCK)-positive neurons. These two interneuron subtypes provide functional dichotomy in the inhibitory network because of their distinct membrane properties, expression patterns of receptors, and their presynaptic modulations (Klausberger et al, 2005; Freund and Katona, 2003). The PVpositive $(\mathrm{PV}+)$ neuron syncytium is regarded as a nonplastic precision clockwork for network oscillations, whereas the CCK-positive $(\mathrm{CCK}+)$ neurons are considered to function as a plastic fine-tuning device that modulates synchronous activities as a function of subcortical inputs (Freund and Katona, 2003). Malfunctioning of the CCK + inhibitory network has been suggested to contribute to emotional disorders including anxiety (Freund and Katona, 2003), although to the best of our knowledge this concept has not been tested experimentally.

We examined how hippocampal GABAergic transmission was altered by the potent synthetic GR agonist dexamethasone (DEX) as well as by acute and chronic restraint stress using whole cell patch-clamp recordings. In addition, we studied the functional dichotomy of PV + and CCK + neurons in the context of chronic stress, specifically the differential effects of chronic stress on PV and CCK interneurons with regard to their ability to generate rhythmic spontaneous IPSCs.

\section{MATERIALS AND METHODS}

\section{Animals and Chronic Restraint Stress}

Adult male Sprague Dawley rats ( $n=48$, Harlan Winkelmann, Borchen, Germany) weighing $170-200 \mathrm{~g}$ at the beginning of the experiment were group housed (3-4 animals/cage). The experiments were performed in accordance with the European Communities Council Directive of 24
November 1986 (86/EEC) and the US National Institutes of Health Guide for the Care and Use of Laboratory Animals, and were approved by the Lower Saxony Federal State Office for Consumer Protection and Food Safety, Germany. Animals were kept under an inverse light cycle (light off from 0700 to 1900 hours), and all experimentation including daily weighing and handling of controls was performed under dimmed red light. Animals of the acute stress group were restrained for $30 \mathrm{~min}$ in well-ventilated polypropylene tubes and decapitated immediately thereafter. During restraint, animals were not physically compressed and did not experience pain. Animals of the chronic stress group were restrained daily for $6 \mathrm{~h}$ without access to food and water (from 0800 to 1400 hours, which is during their active period) for a total of 21 days (McLaughlin et al, 2007). Food was also withheld from the controls during the restraint period to ensure that effects on body weight gain were not simply a result of limited food availability. Throughout the entire experiment, body weight was recorded daily before the onset of restraint. Increased adrenal weight is an indicator of sustained stress. Therefore, in the experiments where brains were perfused for immunocytochemistry, adrenal glands were removed immediately after perfusion and weighed. Adrenal weight was expressed as milligrams per gram of body weight on the last experimental day.

\section{Whole-Cell Recordings}

Whole-cell voltage-clamp recordings were made in acute coronal hippocampal slices ( $350 \mu \mathrm{m}$ thickness) from CA1 pyramidal neurons of the dorsal hippocampus. The bath solution in all experiments consisted of $125 \mathrm{NaCl}, 2.5 \mathrm{KCl}$, $1.25 \mathrm{Na}_{2} \mathrm{HPO}_{4}, 2 \mathrm{MgSO}_{4}, 26 \mathrm{NaHCO}_{3}, 1.5 \mathrm{CaCl}_{2}, 1$ ascorbic

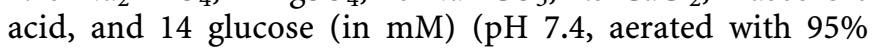
$\mathrm{O}_{2}-5 \% \mathrm{CO}_{2}$ ) and were kept at $30^{\circ} \mathrm{C}$. The pipette solution for all experiments contained $140 \mathrm{KCl}, 1 \mathrm{CaCl}_{2}, 10 \mathrm{EGTA}$, $2 \mathrm{MgCl}_{2}, 0.5 \mathrm{Na}_{2}$-GTP, $4 \mathrm{Na}_{2}$-ATP, and 10 HEPES (in mM); $\mathrm{pH}$ was adjusted to 7.2 with KOH. Spontaneous GABAergic inhibitory postsynaptic currents (sIPSCs) were recorded at a holding potential of $-70 \mathrm{mV}$ in the presence of the $10 \mu \mathrm{M}$ AMPA antagonist 6-cyano-7-nitroquinoxaline-2,3-dione (CNQX), $40 \mu \mathrm{M}$ NMDA antagonist 2-amino-5-phosphonovaleric acid (APV), and $1 \mu \mathrm{M}$ glycine receptor antagonist strychnine. Miniature IPSCs (mIPSCs) were recorded in the presence of $0.5 \mu \mathrm{M}$ tetrodotoxin. Signals with amplitudes at least twofold above the background noise were analyzed. There were no significant differences in noise levels between control and stress animals. Patches with a serial resistance of $>20 \mathrm{M} \Omega$, a membrane resistance of $<0.8 \mathrm{G} \Omega$, or leak currrents of $>150 \mathrm{pA}$ were excluded. The membrane currents were filtered by a four-pole Bessel filter at a corner frequency of $2 \mathrm{kHz}$, and digitized at a sampling rate of $5 \mathrm{kHz}$ using the DigiData 1322A interface (Axon Instruments/ Molecular Devices, Sunnyvale, CA). Data acquisition was performed using commercially available software (pClamp 10.1; Axon Instruments/Molecular Devices). MiniAnalysis 6.0.9 (Synaptosoft, Decatur, GA) was used to perform amplitude and frequency analysis of sIPSCs and mIPSCs. To observe the influence of intracellular $\left[\mathrm{Ca}^{2+}\right]$ on the effect of chronic stress, the slices were incubated in the cell-permeable $\mathrm{Ca}^{2+}$ chelator EGTA-AM $(100 \mu \mathrm{M}$ in the 
recording solution) at room temperature. EGTA-AM was washed off after $30 \mathrm{~min}$ in incubation. For analysis of theta rhythms, power spectrum and autocorrelations were performed in Clampfit 10.1. We calculated a value of 'relative theta power' for each cell by summing the spectral power between 4 and $14 \mathrm{~Hz}$, and dividing this by the total spectral power between 1 and $50 \mathrm{~Hz}$ during $10 \mathrm{~s}$ of sIPSC activity (Karson et al, 2008).

\section{Drugs}

CCK-8, LY225910 were obtained from Tocris. The DEX-BSA conjugate was obtained from Steraloids (Newport, RI). EGTA-AM was obtained from Molecular Probe (Invitrogen, Carlsbad, CA). All other chemicals were obtained from Sigma-Aldrich (St Louis, MO).

\section{Perfusion and Immunocytochemistry}

Control and chronically stressed rats ( $n=6$ /group) were terminally anesthetized with an overdose of ketamine $(50 \mathrm{mg} / \mathrm{ml})$, xylazine $(10 \mathrm{mg} / \mathrm{ml})$, and atropine $(0.1 \mathrm{mg} / \mathrm{ml})$, and perfused transcardially with ice-cold $0.9 \%$ saline, followed by $200 \mathrm{ml}$ of ice-cold fixative containing $4 \%$ paraformaldehyde in $0.1 \mathrm{M}$ sodium-phosphate buffer (PBS, $\mathrm{pH}$ 7.2) for $15 \mathrm{~min}$. The descending aorta was clamped so that adrenal glands were not perfused. To prevent postperfusion artifacts in the brains, the heads were post-fixed overnight in fresh fixative at $4{ }^{\circ} \mathrm{C}$. On the following day, the brains were gently removed, washed thoroughly in PBS, and immersed in $30 \%$ sucrose in $\mathrm{PBS}$ at $4^{\circ} \mathrm{C}$ for $24 \mathrm{~h}$ for cryoprotection. After calibration, brains were frozen and coronal sections $(50 \mu \mathrm{m})$ were cut in a cryostat. Samples from the two groups were processed in parallel to avoid any nonspecific effect of the staining procedure. First, sections were washed in PBS and then treated with $1 \% \mathrm{H}_{2} \mathrm{O}_{2}$ in PBS for $30 \mathrm{~min}$, rinsed three times in PBS, and preincubated in PBS containing 3\% normal goat-serum and $0.5 \%$ Triton $\mathrm{X}-100$. After preincubation, sections were incubated either with a polyclonal rabbit anti-cholecystokinin-8 antibody (AbCam, Cambridge, MA, AB43842, dilution 1:10000) or with a monoclonal mouse anti-parvalbumin antibody (Chemicon/Millipore, Temecula, CA; MAB1572, dilution $1: 3000)$ overnight at $4{ }^{\circ} \mathrm{C}$. The next day, sections were rinsed three times in PBS and then incubated for $2 \mathrm{~h}$ at room temperature either with biotinylated goat anti-rabbit (diluted 1:200; Vector Laboratories, Burlingame, CA) or with biotinylated goat anti-mouse (diluted 1:200, Vector Laboratories) secondary antibody. After washing, the sections were incubated with the avidin-biotin-horseradish peroxidase complex (Vectastain Elite ABC Kit, Vector Laboratories) for $1 \mathrm{~h}$, rinsed again in PBS, developed for $5 \mathrm{~min}$ in diaminobenzidine (DAB Peroxidase Substrate Kit, Vector Laboratories), and then thoroughly rinsed in PBS. The sections were mounted on glass slides in a $0.1 \%$ gelatin solution and dried overnight, after which they were dehydrated through a series of alcohols, cleared in xylene for $30 \mathrm{~min}$ and coverslipped with Eukitt (Kindler, Freiburg, Germany).

\section{Quantification of CCK and PV-Immunoreactive Neurons}

Every sixth section throughout the dorsal hippocampus was examined and found to yield a mean of six sections per animal. Cell numbers are expressed as densities, ie, the number of counted cells was divided by the volume of the analyzed hippocampal area.

\section{Statistical Analysis}

Data are presented as mean \pm SEM. Unless noted, data on drug effects were analyzed by paired-sample Student's $t$-test. Data on the time course of the DEX effect were analyzed by one-way ANOVA followed by Dunnett's Multiple Comparison. Data on stress effect on sIPSCs were analyzed by unpaired $t$-test or ANOVA as indicated in Results section. Data on $\mathrm{PV}+$ and $\mathrm{CCK}+$ cell numbers within the different hippocampal subareas of control and chronically stressed rats were analyzed using one-way ANOVA followed by Tukey's post hoc test.

\section{RESULTS}

\section{The Glucocorticoid DEX Facilitates GABAergic Transmission through a Rapid Non-Classical GR Mechanism}

It is well known that stress affects the function of hippocampal excitatory transmission through glucocorticoid action. Here, we hypothesize that GR activation may also affect the inhibitory network. To directly test this, we applied the potent and selective GR agonist DEX on hippocampal slices of rats. After the application of DEX $(25 \mathrm{nM})$, we detected a rapid increase in sIPSC frequency $\left(6.0 \pm 0.6 \mathrm{~Hz}\right.$ before and $9.2 \pm 0.7 \mathrm{~Hz}$ after DEX, $t_{8}=8.386$, $P<0.0001$, paired $t$-test; Figure $1 \mathrm{a}$ and $\mathrm{c} 1$ ) and amplitude $(98.9 \pm 14.4 \mathrm{pA}$ before and $140.8 \pm 25.5 \mathrm{pA}$ after DEX, $t_{8}=3.084, P=0.015$, Figure $1 \mathrm{a}$ and $\mathrm{c} 2$ ). In all experiments, the initial significant facilitating effect of DEX occurred 5 min after exposure to DEX. This effect accelerated for another few minutes and gradually subsided afterward (Figure 1b1 and b2, one-way ANOVA followed by post hoc Dunnett's Multiple Comparison). In addition, in six of the nine cells recorded, DEX caused burst-like activities that were not detected before DEX application (Figure 1a, arrow in the lower trace). Such a fast DEX effect has not been described before (Maggio and Segal, 2009). By contrast, miniature IPSCs (mIPSCs) showed no significant changes in response to DEX application (frequency: $2.3 \pm 0.3 \mathrm{~Hz}$ before and $2.2 \pm 0.3 \mathrm{~Hz}$ after DEX, $t_{6}=0.6281, P=0.5575$; amplitude: $52.3 \pm 2.8 \mathrm{pA}$ before and $54.0 \pm 2.3 \mathrm{pA}$ after DEX, $t_{6}=0.3932, P=0.7104$, paired $t$-test; Figure $1 \mathrm{~d} 1$ and $\mathrm{d} 2$ ), indicating that in contrast to the previously observed slow effect of DEX (Maggio and Segal, 2009), the rapid effect of DEX that was observed here does not act on the terminals.

This rapid onset of the stimulatory effect of DEX on GABA transmission was quite unexpected. Although it has been reported that the activity of the hippocampal pyramidal neurons can be promoted through a nongenomic corticosterone action (Karst et al, 2005), the observed stimulation of sIPSCs could not be due to an increase in the excitatory drive because all the glutamatergic transmission 
a

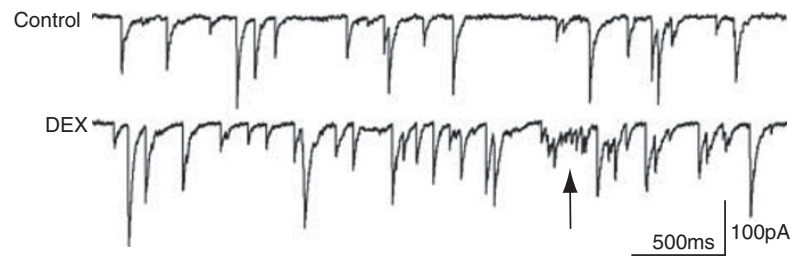

c1

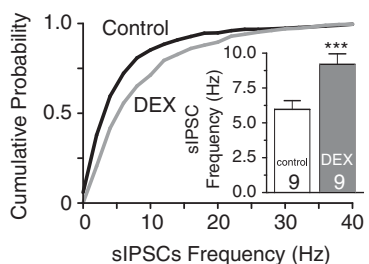

c2

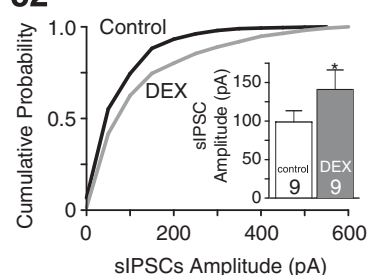

d1

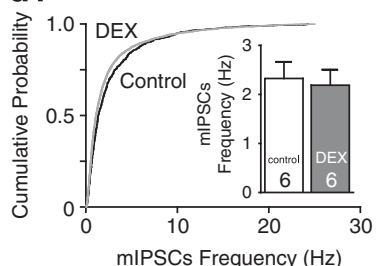

d2

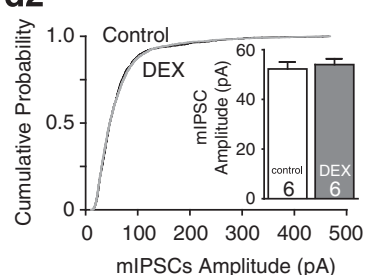

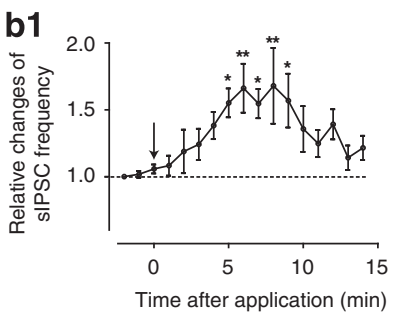

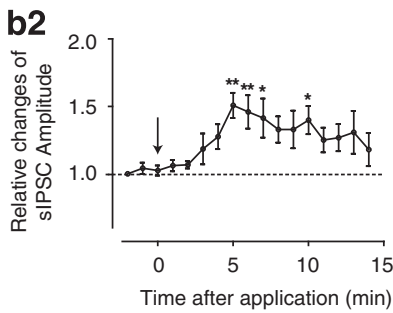

e1

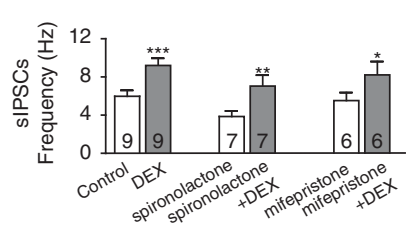

f1

f2

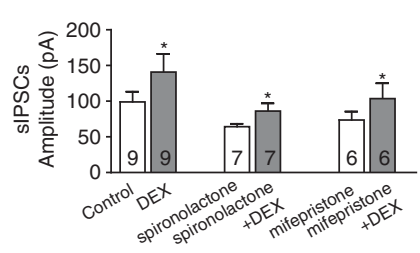

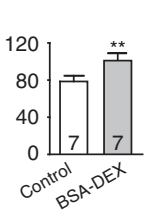

g1

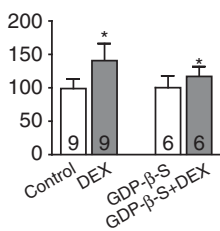

g2

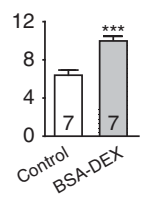

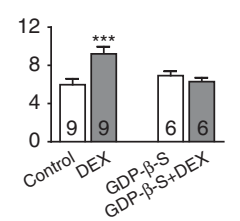

Figure I Glucocorticoid receptor stimulation facilitates GABAergic transmission through a nongenomic mechanism. (a) Representative recordings of sIPSCs in a CAI pyramidal cell before and $\sim 7 \mathrm{~min}$ after the application of the GR agonist DEX, which rapidly increases both the frequency and amplitude of sIPSCs. (b) Time course of DEX effect on sIPSC frequency (b I) and amplitude (b2) (I-min bins; graphs show relative changes). (c) Numerical data of the means and cumulative plot of frequency $(\mathrm{cl})$ and amplitude (c2) of sIPSCs before (white bars) and 5-9 min after DEX (gray bars). (d) Numerical data of the means and cumulative plot of frequency ( $\mathrm{dl}$ ) and amplitude (d2) of mIPSCs before (white bars) and 5-9 min after DEX (gray bars). (e-g) The stimulatory effect of DEX on sIPSCs is mediated by a nongenomic GR. (el, e2) The DEX effect was resistant to the wash-in of spironolactone and mifepristone, which are antagonists at nuclear MRs/GRs. (fI, f2) Membrane-impermeable BSA-DEX could retain the DEX effect. (gl, g2) Intracellular application of GDP- $\beta$-S, which irreversibly inactivates $G$ proteins, blocked the DEX effect. Data are means \pm SEM. Numbers indicate the number of neurons tested for each experimental condition, the same cells in all conditions. Six normal adult male rats were used; $* P<0.05, * * P<0.01, * * * P<0.00$ I.

was blocked in the presence of CNQX and APV (antagonist for AMPA and NMDA receptor, respectively) to isolate sIPSCs. Therefore, we performed a series of experiments to examine the cellular target of DEX. First, the DEX effect described above remained in the presence of the nuclear MR and GR antagonists spironolactone $(10 \mu \mathrm{M})$ (frequency: $3.9 \pm 0.6 \mathrm{~Hz}$ before and $7.0 \pm 1.1 \mathrm{~Hz}$ after DEX, $t_{6}=4.854, P=0.0028$; amplitude: $64.3 \pm 4.6 \mathrm{pA}$ before and $89.1 \pm 12.1 \mathrm{pA}$ after DEX, $t_{6}=2.560, P=0.043$, paired $t$-test; Figure 1e) and mifepristone (10 $\mu \mathrm{M}$; frequency: $5.5 \pm 0.8 \mathrm{~Hz}$ before and $8.2 \pm 1.4 \mathrm{~Hz}$ after DEX, $t_{5}=3.758, P=0.013$; amplitude: $73.6 \pm 11.8 \mathrm{pA}$ before and $103.5 \pm 21.6 \mathrm{pA}$ after $\mathrm{DEX}, t_{5}=2.770, P=0.039$; paired $t$-test; Figure $\left.1 \mathrm{e}\right)$. The antagonists by themselves showed no significant effects on the sIPSCs. Second, bath application of the membraneimpermeable BSA-DEX conjugate $(250 \mathrm{nM})$ retained the stimulatory effect of DEX on GABA release (frequency: $6.4 \pm 0.5 \mathrm{~Hz}$ before and $10.0 \pm 0.5 \mathrm{~Hz}$ after BSA-DEX, $t_{6}=16.59, P<0.0001$; amplitude: $78.5 \pm 6.2 \mathrm{pA}$ before and $101.0 \pm 8.0 \mathrm{pA}$ after BSA-DEX, $t_{6}=4.654, P=0.0035$; paired $t$-test; Figure 1f). Finally, intracellular application of the G-protein inhibitor GDP- $\beta$-S $(0.5 \mathrm{mM})$ through the pipette solution blocked the DEX-induced increase in sIPSC frequency $(6.9 \pm 0.5 \mathrm{~Hz}$ before and $6.3 \pm 0.4 \mathrm{~Hz}$ after DEX, $t_{5}=1.728, P=0.145$; Figure 1g). Taken together, our data strongly suggest that the rapid effect of DEX ( $5 \mathrm{~min}$ after the application) was mediated by a nongenomic membranebound GR that activated a $G$ protein-dependent signaling pathway. This fast mechanism differs from genomic GR-mediated processes ( $>25 \mathrm{~min}$, Maggio and Segal, 2009). Furthermore, as the GDP- $\beta$-S application was restricted to the postsynaptic pyramidal neuron, this result suggests that DEX, at least partially, acts on the postsynaptic cell and that retrograde messenger(s) mediate the stimulatory effect on GABA release.

\section{DEX-Induced Facilitation of GABAergic Transmission is Mediated by Retrograde Nitric Oxide Signaling}

We then examined which retrograde messenger system may be involved in the stimulatory effect of DEX on GABA release. Earlier studies have shown that in the hippocampus, endocannabinoids mediate the activity-dependent suppression of GABAergic inhibition through activation of CB1 receptors that are expressed specifically by $\mathrm{CCK}+$ cells (Freund and Katona, 2003). It is very unlikely that the same retrograde CB1-mediated messenger pathway is responsible for both facilitating and suppressing GABAergic transmission. On the other hand, NO-sensitive guanylyl cyclase has been reported to be present in the axon terminals of both $\mathrm{PV}+$ and CCK + cells (Szabadits et al, 2007). We therefore focused on the retrograde nitric oxide (NO) pathway. 
a $7-\mathrm{NI}$

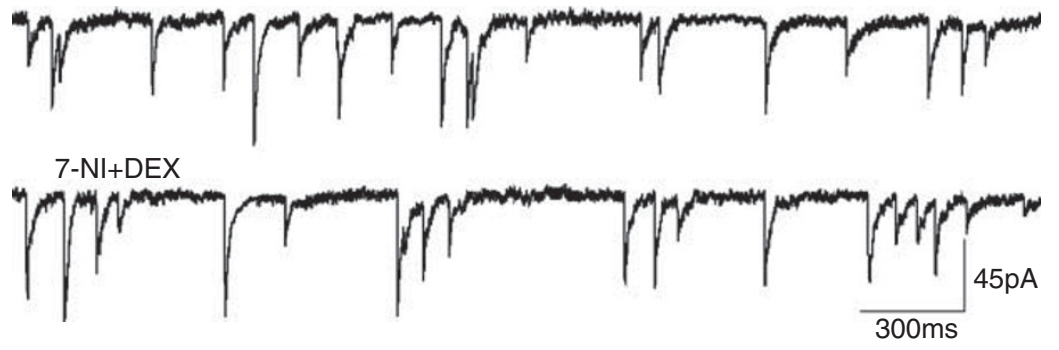

b ODQ

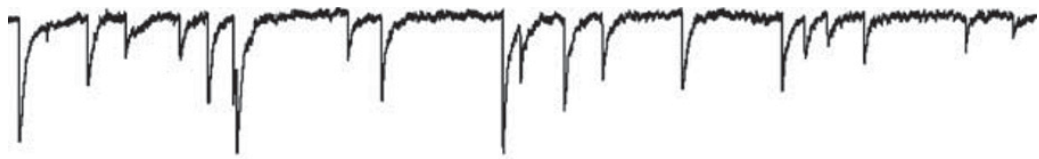

ODQ+DEX

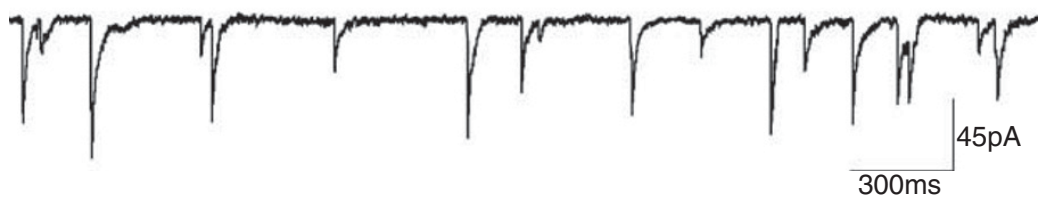

c1 Control

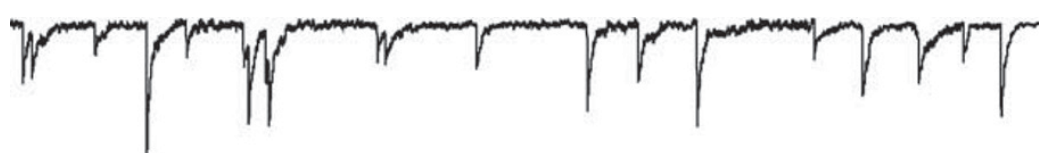

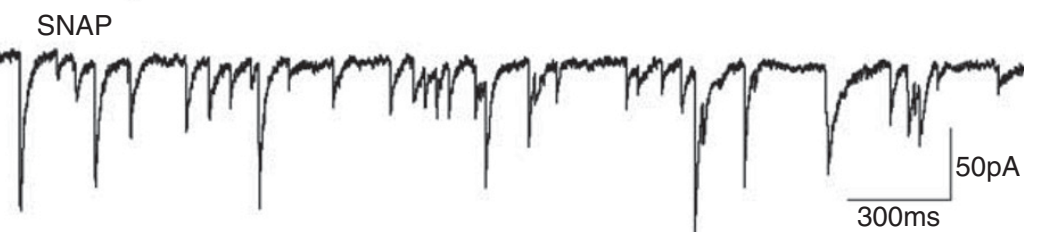

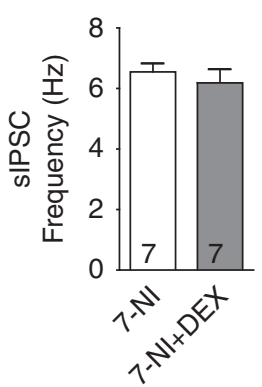
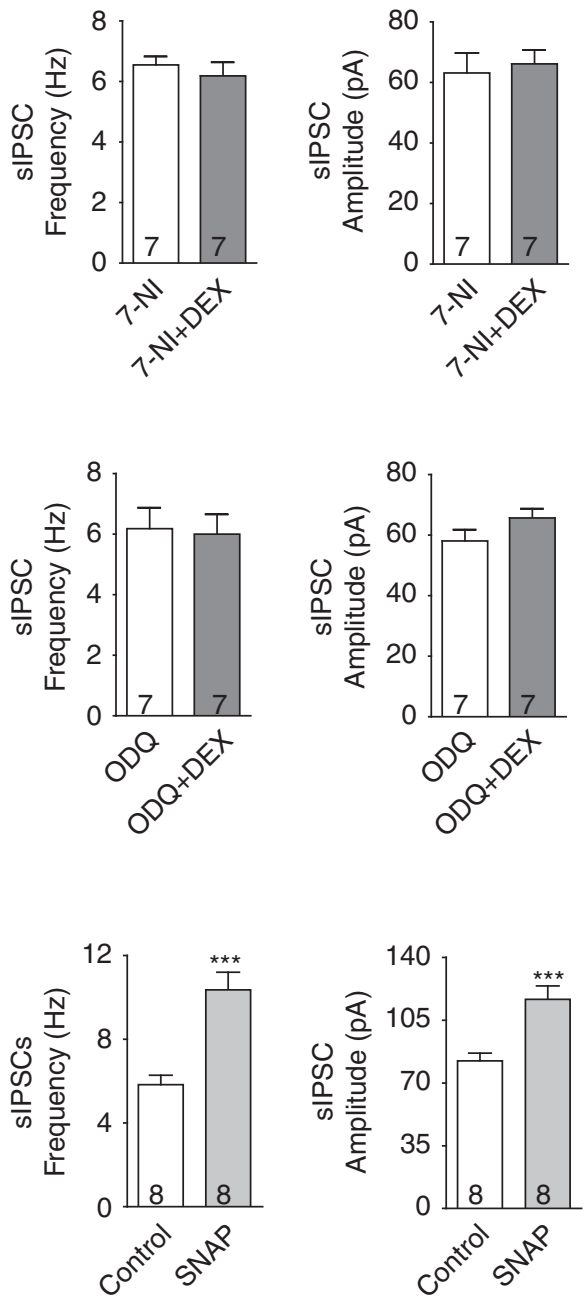

c2

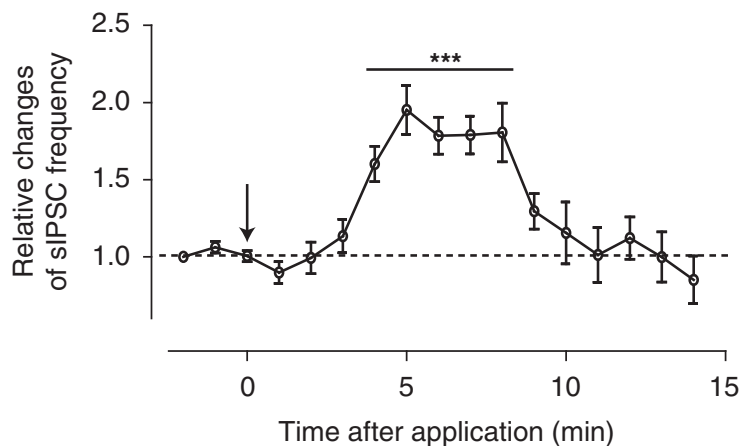

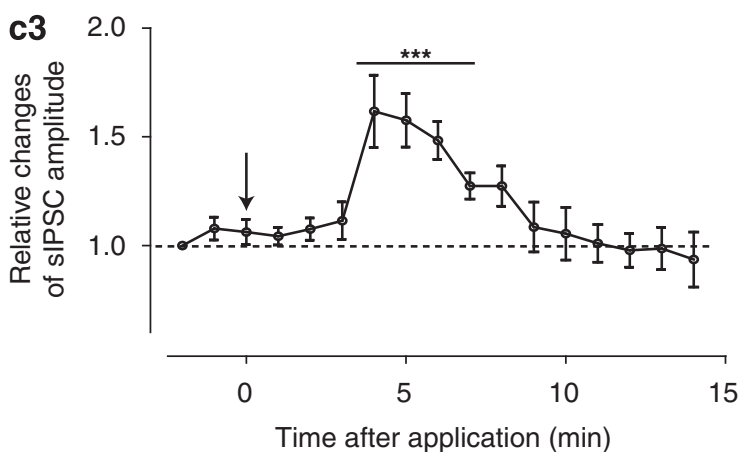

Figure 2 The DEX-induced facilitation of GABA transmission is mediated by the nitric oxide retrograde pathway. (a) The DEX effect is abolished in the presence of an NO synthase inhibitor 7-NI. (Left) Representative recordings of sIPSCs from a CAI pyramidal cell before and $\sim 7$ min after DEX application; 7-NI was applied intracellularly through the pipette solution. (Right) Numerical data of mean sIPSCs before and after DEX. (b) The DEX effect is abolished in the presence of an inhibitor of NO-sensitive guanylyl cyclase, ODQ. (Left) Representative recordings of sIPSCs from a CAI pyramidal cell before and after DEX application; slices were incubated in ODQ for $\sim 30$ min. (Right) Numerical data of mean sIPSCs before and after DEX. (c) Bath application of the NO donor SNAP mimicked the DEX effect. ( $\mathrm{Cl}$ ) left: Representative sIPSC recordings from a CAI pyramidal cell before and $\sim 6$ min after SNAP. (cI) right: Numerical data of mean sIPSCs before and 5-9 min after SNAP. (c2, c3) Time course of the SNAP effect on sIPSCs (I-min bins; graphs show relative changes). Data are mean \pm SEM. Numbers within the bar graphs indicate the number of neurons tested for each experimental condition, the same cells in all conditions; six normal adult male rats were used; **** $P<0.00$ I.

Intracellular application of 7-nitroindazole (7-NI, $100 \mu \mathrm{M})$, a selective inhibitor of neuronal NO synthase, which did not show any significant effect on hippocampal sIPSCs by itself, completely blocked the DEX-induced increase in sIPSC amplitude and frequency (amplitude in 7-NI: $63.1 \pm 6.6 \mathrm{pA}$; amplitude in 7-NI plus DEX: $66.2 \pm 4.6 \mathrm{pA}$; NS; frequency in 7-NI: $6.5 \pm 0.3 \mathrm{~Hz}$; frequency in 7-NI plus DEX: $6.2 \pm 0.5 \mathrm{~Hz}$; NS; Figure 2a). Similarly, 
a 30 min incubation of the slices with ODQ ( $50 \mu \mathrm{M}$ in aCSF), a selective inhibitor of NO-sensitive guanylyl cyclase (NOsGC), completely blocked the effect of DEX on GABA release (sIPSC amplitude in ODQ: $58.1 \pm 3.7 \mathrm{pA}$, amplitude in ODQ plus DEX: $65.7 \pm 3.1 \mathrm{pA}$, NS; frequency in ODQ: $6.2 \pm 0.7 \mathrm{~Hz}$, frequency in ODQ plus DEX: $6.0 \pm 0.6 \mathrm{~Hz}$, NS; Figure 2b). However, incubation with ODQ caused a small reduction in the sIPSC amplitude $\left(\mathrm{t}_{14}=2.418, P=0.0298\right.$; unpaired $t$-test) but not in the frequency, which is most likely due to weak activation of NOsGC at baseline level. These results show that inhibiting NO synthesis or silencing NO downstream signaling can completely block the fast DEX effect mentioned above. In addition, the NO donor SNAP ( $S$-nitroso- $N$-acetylpenicillamine) mimicked the facilitating effect of DEX on sIPCSs. Similar to the effect elicited by DEX, bath application of SNAP $(100 \mu \mathrm{M})$ increased sIPSC amplitude and frequency (baseline amplitude: $82.3 \pm 4.4 \mathrm{pA}$; amplitude after SNAP $116.7 \pm 7.4 \mathrm{pA}$; $P<0.005$; baseline frequency: $5.8 \pm 0.4 \mathrm{~Hz}$; frequency after SNAP $10.4 \pm 0.8 \mathrm{~Hz} ; P<0.005$; paired $t$-test; Figure $2 \mathrm{c}$ ). Furthermore, SNAP $(100 \mu \mathrm{M})$ also mimicked the time course of the fast DEX effect (c.f. Figure $2 \mathrm{c} 2 / \mathrm{c} 3$ to Figure $1 \mathrm{~b} 1 / \mathrm{b} 2)$. Taken together, these results indicate that the fast DEX-induced facilitating effect on GABA transmission is mediated by NO retrograde signaling.

\section{DEX-Induced Facilitation of GABAergic Transmission is Partially Mediated by NO-Induced Release of CCK}

There is substantial evidence that the neuropeptide CCK is involved in stress response (Becker et al, 2008; Hebb et al, 2005). Therefore, we tested whether the enhanced inhibitory activities in the presence of DEX would induce CCK release from CCK interneurons, which would further modulate perisomatic GABA release (Földy et al, 2007). We incubated the slices with the selective $\mathrm{CCK}_{2}$ receptor antagonist LY225910 $(20 \mu \mathrm{M}$ in aCSF) for $30 \mathrm{~min}$ before recording. The antagonist by itself had no effect on sIPSCs, but it blocked the response to further CCK application (data not shown). Then, we applied $25 \mathrm{nM}$ DEX and observed an increase in sIPSC amplitude and frequency (amplitude in LY225910: $79.6 \pm 9.8 \mathrm{pA}$, amplitude in LY225910 plus DEX: $89.8 \pm 7.0 \mathrm{pA}, t_{7}=2.504, P=0.04$; frequency in LY225910: $6.2 \pm 0.9 \mathrm{pA}$, frequency in LY225910 plus DEX: $7.3 \pm 0.8 \mathrm{pA}$, $t_{7}=4.788, P=0.002$; paired $t$-test; Figure $3 \mathrm{a}$ and $\mathrm{c} 1$ ). However, compared with the condition without LY225910 pre-incubation, the extent of the increase was much lower (increase of amplitude: $19.5 \pm 6.0$ vs $40.8 \pm 8.8 \%$, unpaired $t$-test $t_{15}=1.946, P=0.07$; increase of frequency: $24.4 \pm 8.3$ vs $60.2 \pm 9.9 \%$, unpaired $t$-test $t_{15}=2.733, P=0.015$; Figure $3 c 2$ ). It is interesting to note that the duration of fast DEX effect was shortened in the presence of LY225910 (3 vs $5 \mathrm{~min}$; Figure $3 \mathrm{~b}$ ), whereas the onset time was unaffected (one-way ANOVA with Dunnett's multiple comparison test, Figure 3b). Furthermore, after the incubation with LY225910, the stimulatory effect of the NO donor SNAP on GABAergic transmission was also significantly reduced (increase of amplitude: $23.9 \pm 5.1$ vs $42.0 \pm 4.9 \%$, unpaired $t$-test, $t_{14}=2.550, P=0.02$; increase of frequency: $20.8 \pm 3.1$ vs $78.3 \pm 9.6 \%$, unpaired $t$-test, $t_{14}=5.718, P<0.0001$; Figure $3 \mathrm{~d}$ and e). The fact that the $\mathrm{CCK}_{2}$ receptor antagonist partially blocked the fast effects of DEX and SNAP suggests that CCK additionally contributes to DEX action and that NO signaling induces endogenous CCK release, which in turn further facilitates GABAergic transmission.

\section{Acute Stress Enhances Hippocampal GABAergic Transmission}

The above data show that the acute application of DEX caused a rapid facilitation of GABAergic transmission in the hippocampal CA1 area through a nongenomic GR mechanism. As glucocorticoids mediate not only the stress response, it is important to ask whether exposure to real life stress also elicits the enhancement of inhibitory transmission. To answer this, we performed a series of experiments with rats exposed to short-term (acute) restraint stress.

Immediately after exposing animals to $30 \mathrm{~min}$ restraint stress, we found an increase in sIPSC frequency in the hippocampal slices of stressed rats compared with controls (control: $7.3 \pm 0.3 \mathrm{~Hz}$; acute stress: $11.8 \pm 0.7 \mathrm{~Hz} ; t_{15}=5.424$ $P<0.0001$; unpaired $t$-test; Figure $4 \mathrm{a}$ and $\mathrm{b} 1)$, whereas the amplitude of the sIPSCs remained unaffected (control: $90.01 \pm 9.3 \mathrm{pA}$; acute stress: $98.41 \pm 11.2 \mathrm{pA} ; t_{15}=0.5772$ $P=0.5723$; Figure $4 \mathrm{a}$ and $\mathrm{b} 2$ ). In addition, in 7 out of the 10 cells recorded from the acutely stressed animals, burstlike activities were frequently observed, whereas in the control group, this phenomenon was sparsely detected (2 of the 7 cells, data not shown). These data show that acute stress exposure results in an enhancement in hippocampal GABAergic transmission that is similar though not identical to the effect elicited by DEX (see Figure 1 and Maggio and Segal, 2009).

Figure 3 The DEX-induced increase in GABAergic transmission is partially mediated by endogenous CCK release downstream from the nitric oxide signaling. (a) Representative sIPSCs from a CAI pyramidal cell after incubation with the CCK 2 receptor antagonist LY2259I0 for 30 min and then $~ 7$ min after DEX application. (b) Time course of the DEX effect following 30 min-incubation with LY2259 I0 (I -min bins; graphs show relative changes), showing that the duration of significant DEX effect was shortened by blocking $\mathrm{CCK}_{2}$ receptor. (c) Numerical data of mean sIPSCs before and 5-7 min after DEX exposure in the presence of LY2259I0 (cI) and mean relative changes of sIPSCs (c2) in response to DEX without and with LY2259I0, respectively. Bath application of DEX still induced a significant increase in SIPSCs. However, the increase was smaller compared with the condition without LY2259I0 preincubation, revealing that CCK-mediated signaling is also involved in the DEX-induced enhancement of sIPSCs. (d) Representative sIPSCs after bath application of the NO donor SNAP in the presence of LY2259I0. (e) Numerical data of mean sIPSCs (el) and mean relative changes of sIPSCs (e2) in response to SNAP without and with LY2259I0, respectively, showing that LY2259I0 partially blocked the stimulatory effect of SNAP. This indicates that NO signaling induces endogenous CCK release, which in turn facilitates GABAergic transmission. Data are mean \pm SEM. Numbers within the bar graphs indicate the number of neurons tested for each experimental condition, same cells in all conditions; seven normal adult male rats were used; *P $<0.05$, $* * P<0.01, * * * P<0.001$. 
a

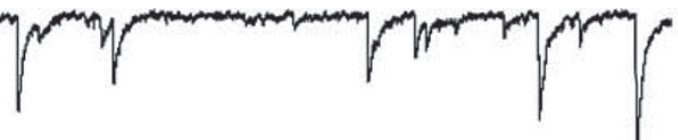

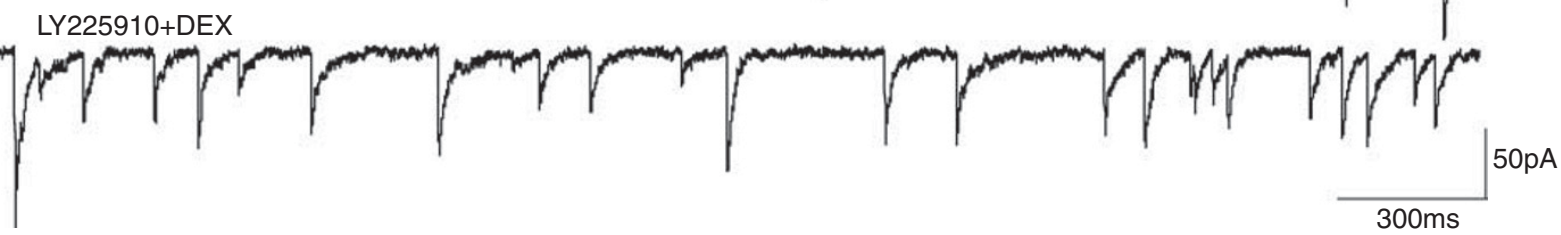

b1

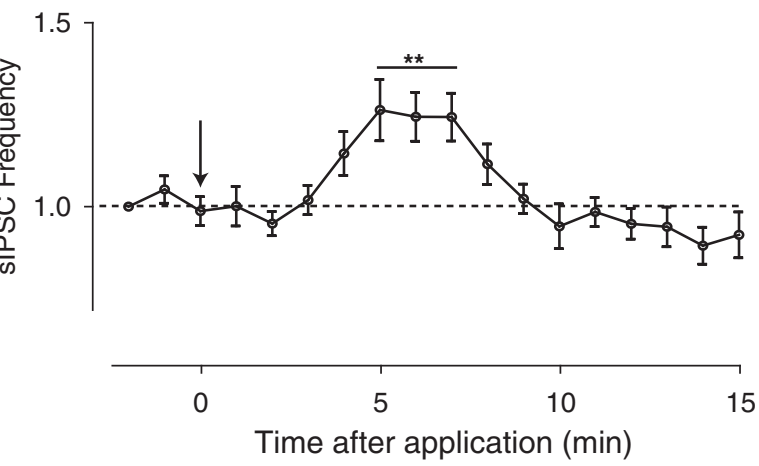

c1

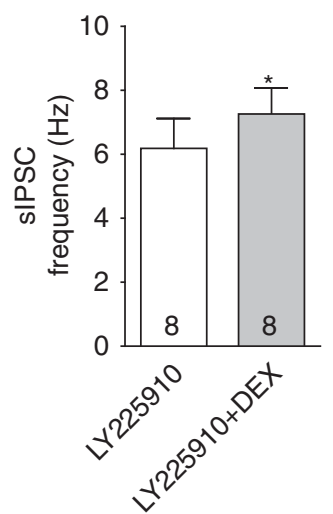

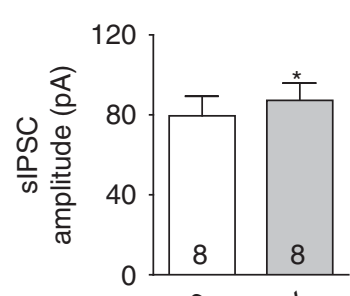

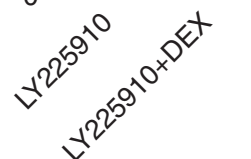

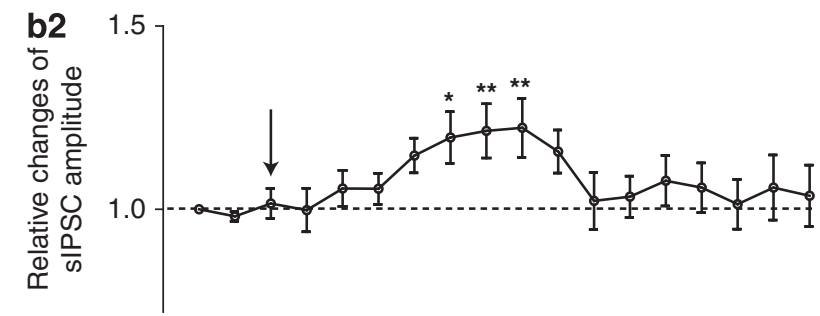
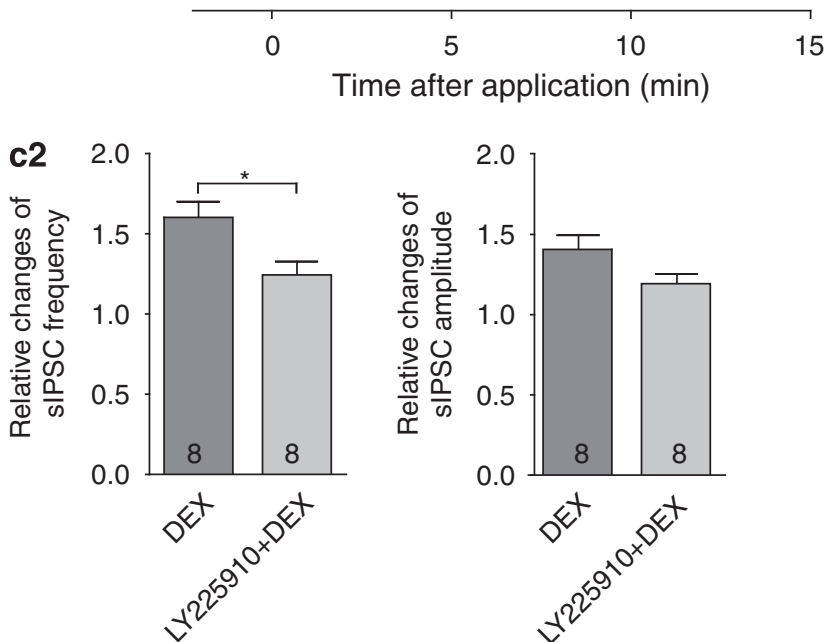

d LY225910+SNAP

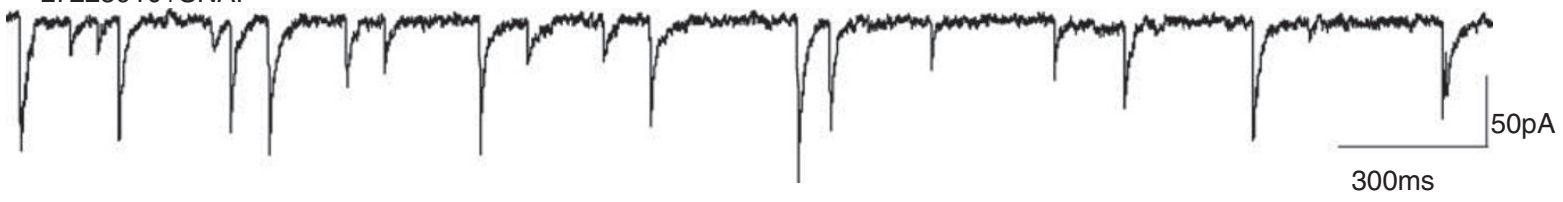

e1

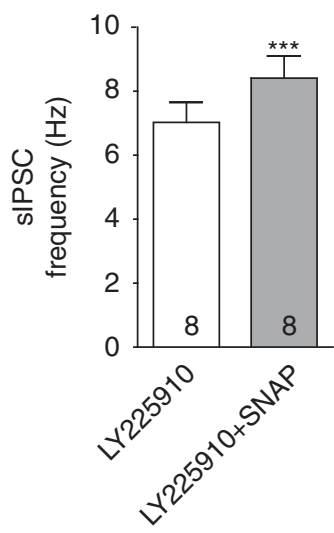

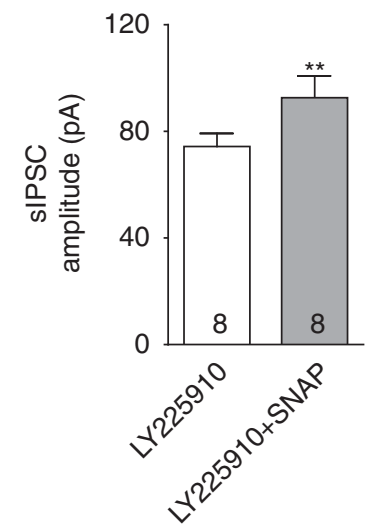

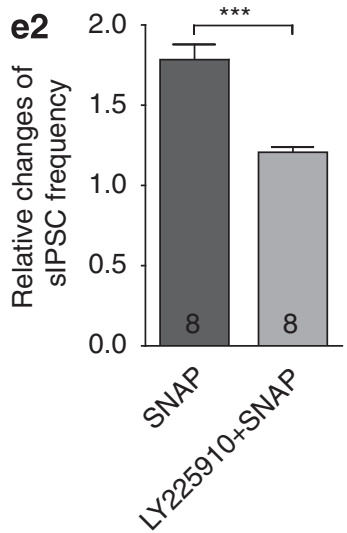

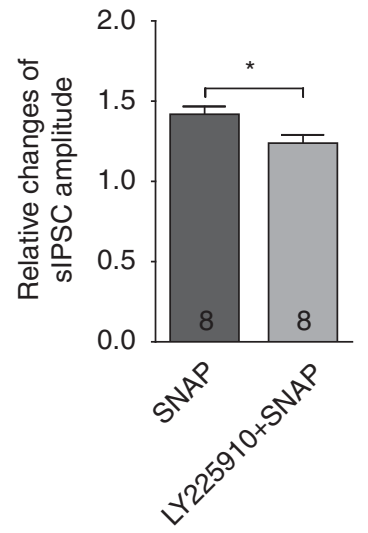



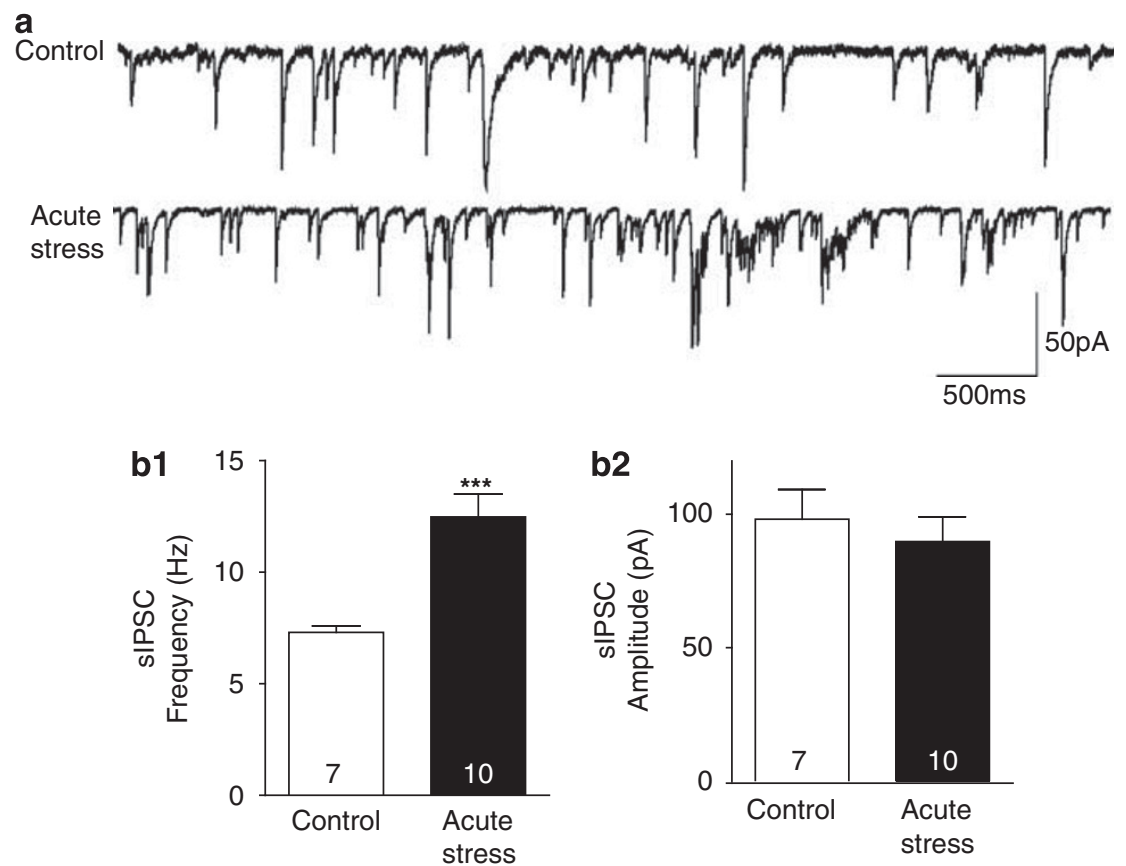

Figure 4 Acute stress increases hippocampal GABAergic transmission. (a) Representative voltage-clamp recordings of sIPSCs in CAI pyramidal cells of control and acutely stressed rats. Acute stress significantly increased sIPSC frequency (bI), whereas the amplitude (b2) was not affected. Data are mean \pm SEM; numbers within the bars indicate the number of neurons recorded; $* * * P<0.001$.

\section{Chronic Stress Results in a $\mathrm{Ca}^{2+}$-Dependent Elevation of GABAergic Transmission}

We then asked whether the elevation in hippocampal sIPSCs sustains after long-term stress during which the system is exposed to a prolonged high level of stress hormones including corticosterone. To examine this, we used rats exposed to 3 weeks of restraint stress. To assess the physiological effects of chronic restraint stress, body weights were recorded daily throughout the experiment and adrenal weights were determined at the end of the experiment. Stress significantly reduced body weight gain, and two-way ANOVA (stress $\times$ time) revealed a significant main effect of stress $\left(\mathrm{F}_{1,27}=606.6, P<0.001\right.$; Figure $\left.5 \mathrm{a}\right)$. Furthermore, chronic stress increased the relative adrenal weights (two-tailed unpaired $t$-test: $t_{10}=2.579, P<0.05$; Figure 5b), which is in line with earlier findings that increased adrenal weights are reliable indicators of sustained HPA (hypothalamus-pituitary-adrenal) axis hyperactivity (Magariños and McEwen, 1995; McLaughlin et al, 2007).

We next investigated whether long-term stress affected the occurrence of sIPSCs in CA1 pyramidal neurons. Exposure to chronic restraint stress resulted in a significant increase in the frequency of hippocampal sIPSCs (control: $5.9 \pm 0.3 \mathrm{~Hz}$; stress: $10.4 \pm 0.5 \mathrm{~Hz} ; t_{48}=9.279 ; \quad P<0.0001$; Figure $6 \mathrm{a}$ and $\mathrm{b}$ ), without significant changes in the amplitude of the sIPSCs (control: $96.1 \pm 6.6 \mathrm{pA}$; stress: $97.2 \pm 8.1 \mathrm{pA}$; NS; Figure $6 \mathrm{a}$ and c). Burst-like activities were also observed in 10 of 16 cells recorded from this group.

Furthermore, stress-induced enhancement of the sIPSC frequency could be normalized by the membranepermeable $\mathrm{Ca}^{2+}$ chelator EGTA-AM, which reduces the concentration of intracellular free $\mathrm{Ca}^{2+}$. One-way ANOVA revealed a significant difference in the sIPSC frequency between the groups $\left(\mathrm{F}_{(3,60)}=35.19 ; P<0.0001\right)$. Tukey's post hoc test showed a significant difference between the control and the acute stress groups $(q=13.05 ; P<0.001)$. Bath application of EGTA-AM to the hippocampal slices of chronically stressed animals normalized the sIPSC frequency to control level $(100 \mu \mathrm{M}$; stress + EGTA-AM: $6.4 \pm 0.4 \mathrm{~Hz} ; \quad q=7.884, \quad P<0.001 \quad v s$ stress; $q=1.208$, $P>0.05$ vs control; Figure $6 a, b$, and $c$ ), whereas in the control slices, EGTA-AM caused no significant changes in sIPSCs (Figure $6 \mathrm{a}, \mathrm{b}$, and $\mathrm{c}$ ). This indicates that chronic stress induced an enhancement in GABAergic transmission, which is concomitant with an increased intracellular-free $\mathrm{Ca}^{2+}$ in hippocampal interneurons.

Importantly, bath application of DEX ( $25 \mathrm{nM})$ to the slices from the chronically stressed rats failed to elicit any significant change in sIPSCs of the CA1 pyramidal neurons (frequency: $10.2 \pm 0.9 \mathrm{~Hz}$ before and $10.3 \pm 1.0 \mathrm{~Hz}$ after DEX, paired $t$-test, NS; amplitude: $87.2 \pm 6.4 \mathrm{pA}$ before and $85.3 \pm 5.1 \mathrm{pA}$ after DEX, paired $t$-test, NS, Figure 7). The origin of the loss of the DEX effect after chronic stress is yet to be determined (see Discussion section).

\section{Chronic Stress Reduces the Number of the} PV-Immunoreactive Neurons, whereas the Number of CCK-Immunoreactive Neurons Remains Unchanged

Considering (i) the theory of functional dichotomy of PV + and CCK + neurons proposed by Freund (Freund and Katona, 2003) and (ii) our present results that indicate that DEX facilitates the release of endogenous CCK (Figure 3), which specifically stimulates $\mathrm{PV}+$ neurons, we asked 


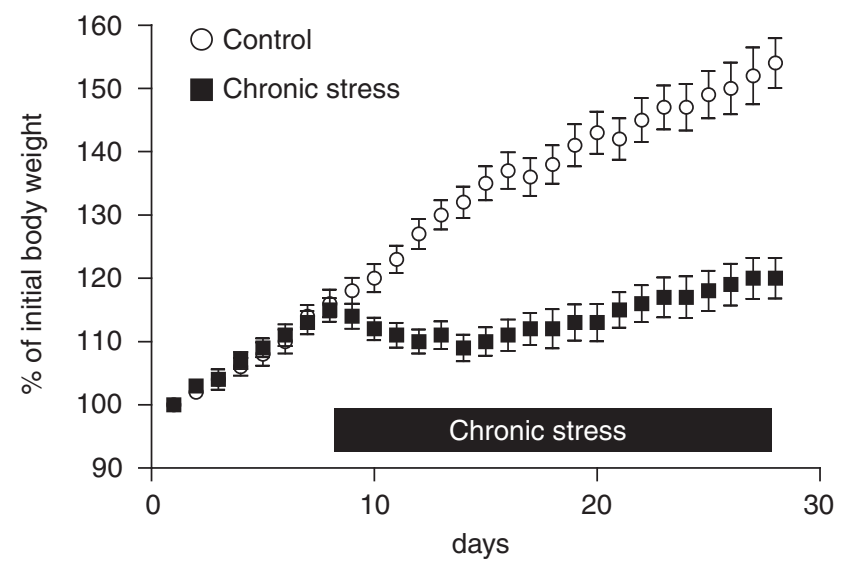

b

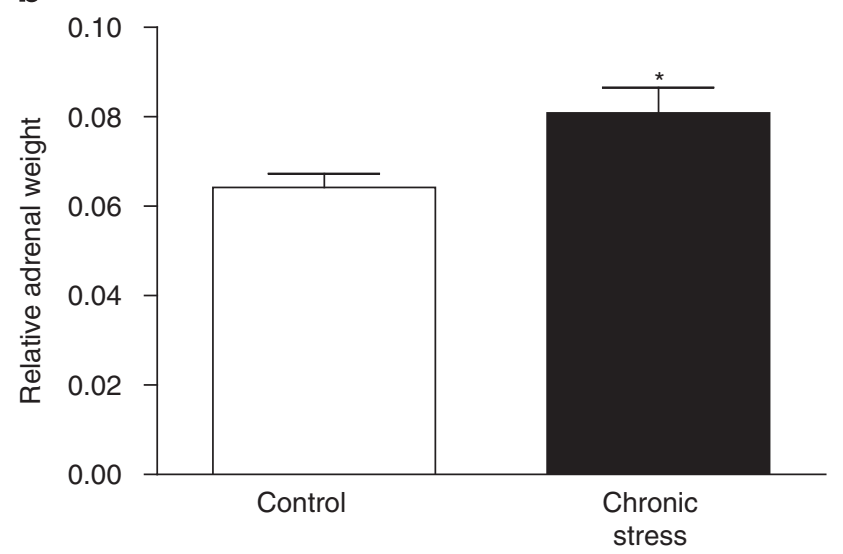

Figure 5 Physiological parameters showing the responses to chronic stress. (a) Body weight gain (control: $n=23$; Stress: $n=22$ ), (b) Relative weight of the adrenal glands (mg per $100 \mathrm{~g}$ body weight, six animals per group). Data are mean \pm SEM. $* P<0.05$. whether these two subtypes of perisomatic interneurons are differentially affected by chronic stress. We first examined the effects of chronic restraint stress on the structural integrity of $\mathrm{PV}+$ and $\mathrm{CCK}+$ neurons. A number of these neurons in the dorsal hippocampus of control and chronically stressed rats were quantified. We found that chronic stress decreased the number of PV-immunoreactive neurons in all hippocampal subregions: $31 \%$ reduction in dentate gyrus $(P<0.01$, Figure $8 \mathrm{c}), 23 \%$ reduction in CA2-3 $(P<0.01$, Figure $8 \mathrm{c})$, and $36 \%$ reduction in CA1 $(P<0.01$, Figure 8a1, a2, and c). In contrast, the number of CCKimmunoreactive neurons remained unaltered (Figure 8b1, b2, and d).

\section{Chronic Stress Impairs CCK-Induced Rhythmic Burst Activity Originating from the PV Interneurons}

We then ask whether the above differential effects of chronic stress on the number of PV- and CCK-immunoreactive neurons also affect the function of PV + and CCK + neurons. It is reported that an elaborately regulated $\mathrm{Ca}^{2+}$ signaling is essential for the temporal precision of the output from interneurons (Hefft and Jonas, 2005). As our data indicated that chronic stress induced an excessive intracellular free $\mathrm{Ca}^{2+}$ in the hippocampal interneurons, we decided to test whether chronic stress affects the ability of the perisomatic interneurons to generate rhythmic firing, which is of great importance in controlling the timing of pyramidal neuron action potentials. It is known that in the hippocampus, both CCK analogs and the muscarinic acetylcholine receptor agonist carbachol can provoke rhythmic sIPSCs in vitro. Carbachol-induced rhythmic IPSCs are inhibited by endocannabinoids, by Ntype calcium channel blockers, and by activation of $\mathrm{GABA}_{\mathrm{B}}$ receptors (Karson et al, 2008). In contrast, CCK-triggered IPSCs are inhibited by P/Q-type calcium channel blockers, but are insensitive to endocannabinoids (Karson et al,

a

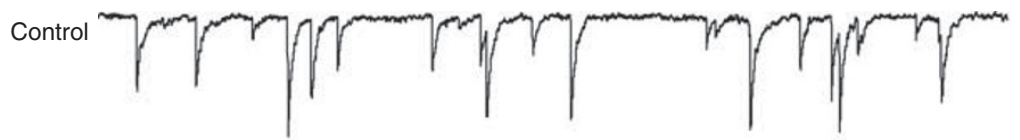

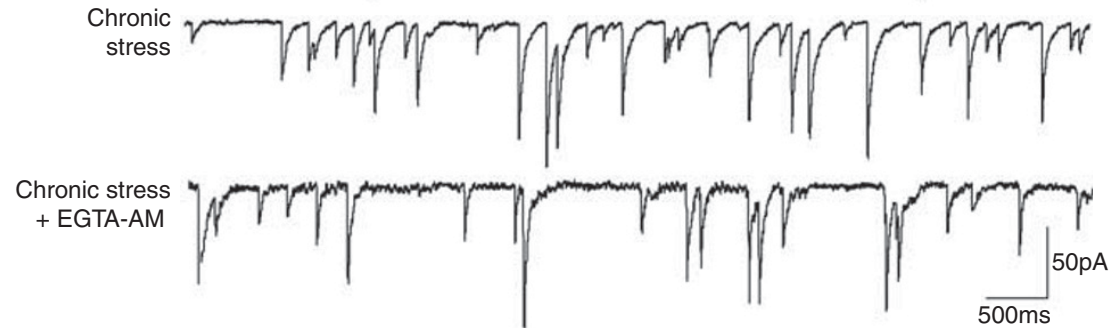

b
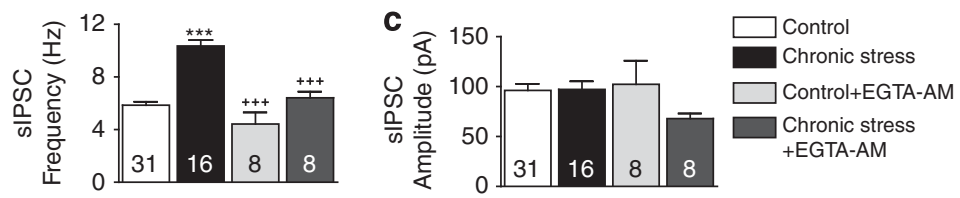

Figure 6 Chronic stress increases hippocampal GABAergic transmission. (a) Representative voltage-clamp recordings of sIPSCs in CAI pyramidal cells of a control and a chronically stressed rat. Stress significantly increased sIPSC frequency (b), whereas the amplitude (c) was not affected (control: $n=\mid 4$; stress: $n=12$ ). The sIPSC frequency, which was elevated by stress, was normalized by EGTA-AM (seven animals per group). Data are mean \pm SEM; numbers within the bars indicate the number of neurons recorded; ${ }^{* * *} P<0.00$ I, vs control, ${ }^{++}+P<0.00$ I, vs stress. 
a
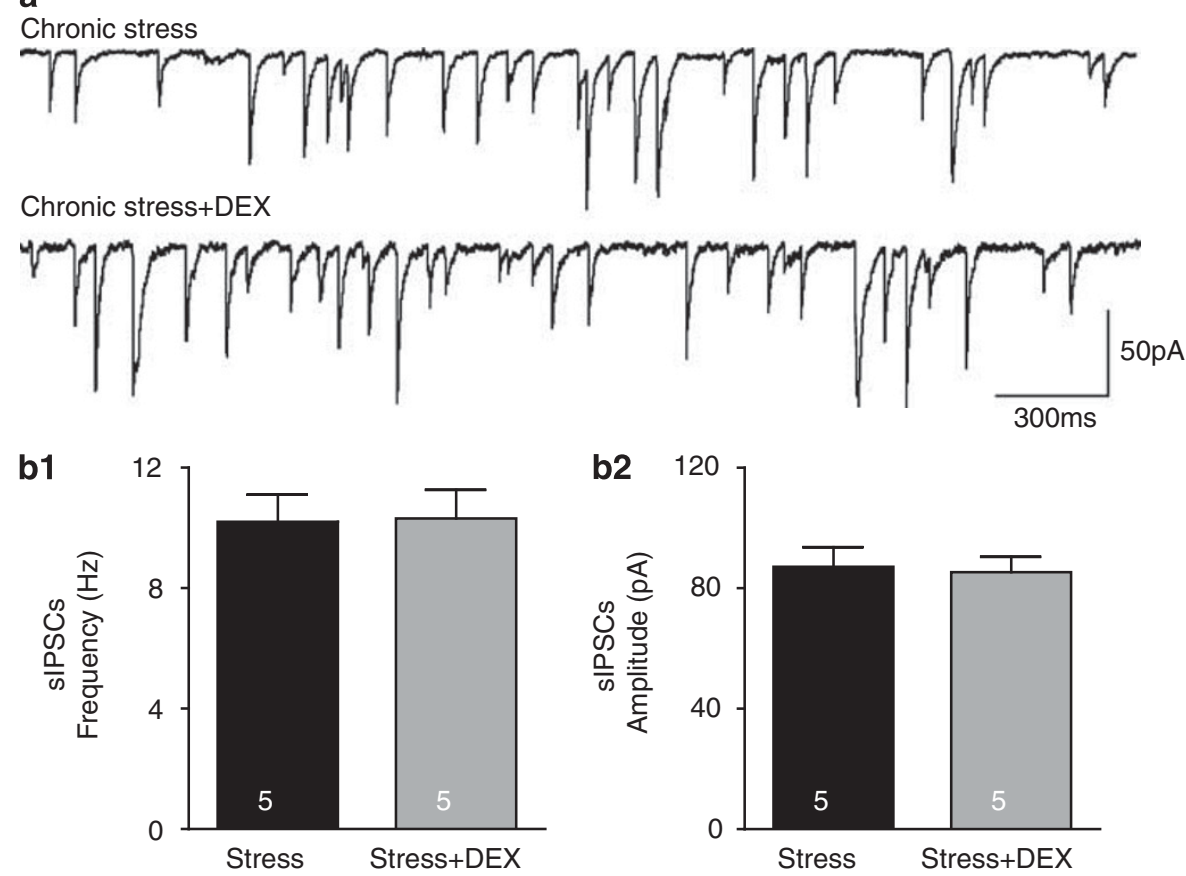

Figure 7 Bath application of DEX $(25 \mathrm{nM})$ failed to elicit any significant change in sIPSCs in the hippocampus of chronically stressed rats. (a) Representative recordings of sIPSCs in a CAI pyramidal cell from a stressed rat before and after the application of the GR agonist DEX. (b) Numerical data of the means of frequency (bl) and amplitude (b2) of sIPSCs before (black bar) and after DEX (gray bar) (five neurons recorded from five stressed animals). Data are mean \pm SEM.

2008). Given the distinct cellular properties of CCK + and $\mathrm{PV}+$ neurons, carbachol presumably triggers rhythmic sIPSCs originating from CCK + cells, whereas CCK stimulates sIPSCs from PV + cells (Karson et al, 2008). In this study, the bath application of carbachol $(5 \mu \mathrm{M})$ induced a rhythmic pattern of sIPSCs in hippocampal slices of control rats (Figure 9a1 and a2). Autocorrelation analysis of 10 -s stretches of sIPSCs (selected from shortly after the onset of the enhancement) in all 8 cells showed regularrepeating peaks. After carbachol application, power spectral analysis of the same stretches from each cell revealed a significant enhancement in the total power and, more importantly, a sharp peak power within the theta frequency range, $4-14 \mathrm{~Hz}$ (relative theta power was $46.7 \pm 1.1 \%$ before carbachol and $57.7 \pm 1.1 \%$ after carbachol; $t_{7}=8.326$, $P<0.0001$; paired $t$-test; Figure 9a2 and e). Similarly, the bath application of the CCK analog CCK8-S $(1 \mu \mathrm{M})$ induced rhythmic sIPSCs that were manifested by regular-repeating peaks in the autocorrelation analysis, and a significant increase of power specifically in the theta frequency range in all eight tested cells (relative theta power was $47.1 \pm 1.0 \%$ before and $60.2 \pm 1.9 \%$ after CCK application; $t_{7}=6.374$, $P<0.0001$; paired $t$-test; Figure 9c1, c2, and e). Consistent with previous studies, the rhythmicity triggered by the CCK analog was less variable and more persistent compared with that induced by carbachol.

In hippocampal slices from chronically stressed rats, the response to carbachol was similar to that in controls (relative theta power before carbachol: $46.2 \pm 1.5 \%$, after carbachol: $59.4 \pm 1.9 \% ; t_{7}=12.28, P<0.0001$; Figure $9 \mathrm{~b} 1, \mathrm{~b} 2$, and e). Two-way ANOVA of repeated measures on data from the control and the chronic stress groups also revealed no significant effect of stress $(\mathrm{F}=0.1567 ; P=0.6991)$. However, CCK8-S failed to induce rhythmicity in all 10 tested cells. In contrast to control, the CCK agonist induced an arrhythmic pattern in the slices from stressed rats as indicated by flat autocorrelation curves. Similarly, CCK8-S did not change relative theta power in stressed rats (before CCK $49.4 \pm 1.2 \%$; after CCK $48.7 \pm 1.6 \%$; $t_{9}=0.730, P=0.48$; Figure $9 \mathrm{~d} 1, \mathrm{~d} 2$, and e) despite a massive increase in the total power. This shows that in addition to the enhancement of GABAergic transmission, the temporal precision in firing of the PV + cells is impaired after chronic stress, whereas the same parameter remains unaffected in CCK + cells.

\section{DISCUSSION}

This study provides four novel findings: (1) acute application of a GR agonist rapidly elicits an increase in sIPSCs in the hippocampus, notably through a membrane-bound GR and by retrograde NO signaling; (2) exposing animals to a brief acute stress causes a similar enhancement of hippocampal sIPSCs; (3) chronic stress also results in elevated GABAergic transmission in the hippocampus, which is $\mathrm{Ca}^{2+}$-dependent, and DEX application elicits no further increase in sIPSCs; (4) chronic stress impairs the generation of rhythmic spontaneous IPSCs originating specifically from PV + neurons. Given the pivotal role of perisomatic inhibition in synchronizing activities of pyramidal neurons, the chronic stress-induced deficit of the PV interneurons to generate rhythmic firing may result in abnormal network oscillations and thus contribute to cognitive impairments that are often observed in patients 

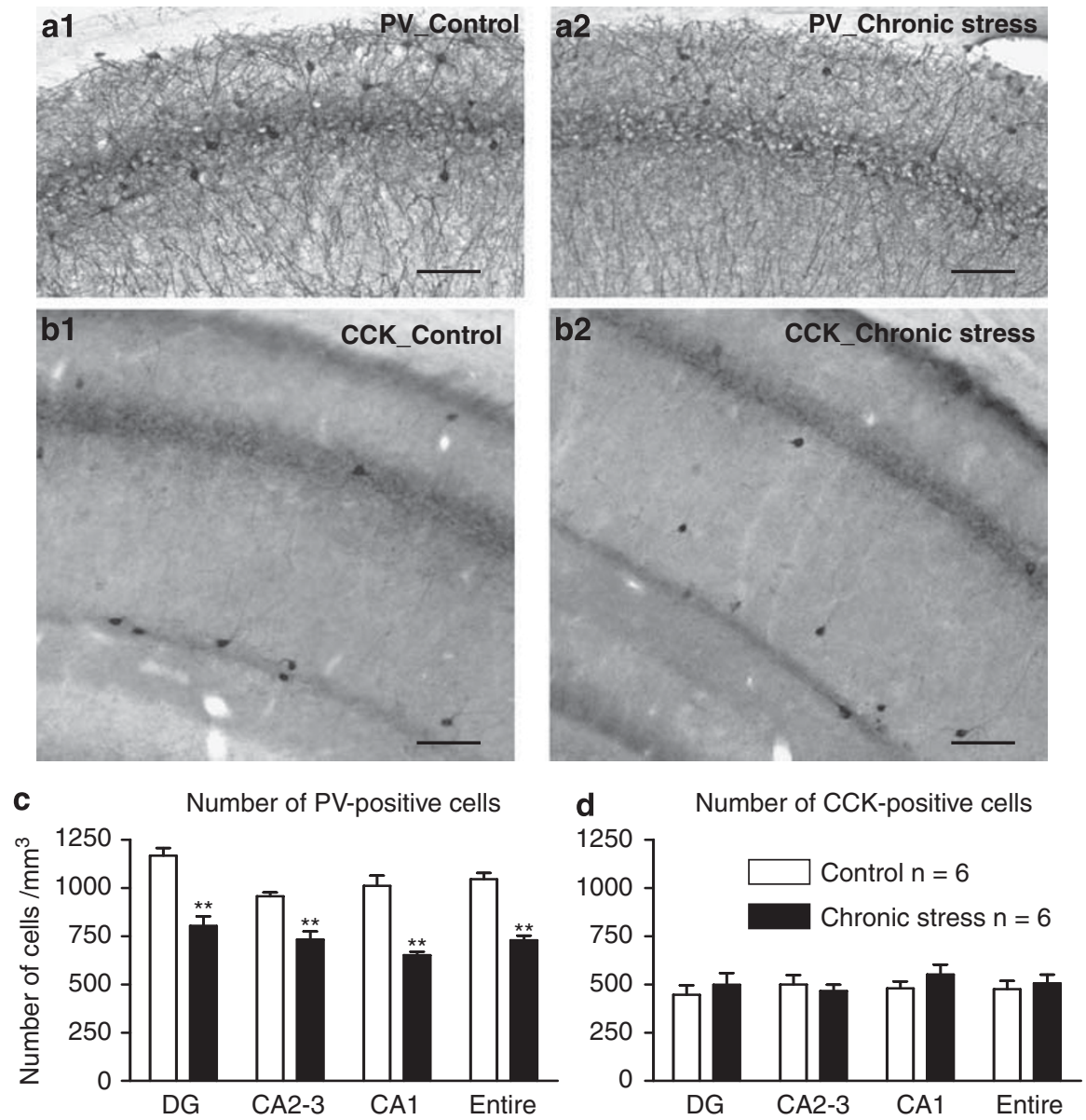

Figure 8 Chronic stress reduces the number of PV-immunoreactive cells, whereas the number of CCK-immunoreactive cells remains unaltered. (a) Representative examples of parvalbumin-stained CAI sections from a control (al) and a stressed rat (a2). (b) Representative examples of cholecystokininstained CAI sections from a control (bl) and a stressed (b2) rat. Stress significantly decreased the number of PV + cells in all hippocampal subareas (c), whereas the number of CCK + cells was not affected (d). This indicates that chronic stress affects the structural integrity of PV + but not of CCK + cells. Data are mean \pm SEM; six animals per group were analyzed; $* * P<0.0$ I. DG, dentate gyrus. Scale bars: $100 \mu \mathrm{m}$.

with stress-related psychiatric disorders. An association between the functional deficit of PV interneurons and the altered $\gamma$-oscillation has been suggested as an underlying mechanism in working memory deficits in schizophrenic patients (Lewis et al, 2005; Lodge et al, 2009).

\section{Glucocorticoids Facilitate GABAergic Transmission by Activating Nongenomic GRs}

Stress activates the HPA axis to increase release of corticosteroids from the adrenal glands. The cellular effects of corticosteroids are mediated chiefly through slow genomic mechanisms (De Kloet et al, 1998; Maggio and Segal, 2009). However, the existence of a rapid nongenomic pathway recently gained substantial credit (Di et al, 2005, 2009; Haller et al, 2008; Karst et al, 2005; Campolongo et al, 2009). In the hypothalamus, corticosterone elicits rapid opposing effects on glutamate and GABA release of magnocellular neurons through retrograde endocannabinoid and NO signaling through divergent nongenomic pathways (Di et al, 2005, 2009). In the basolateral nucleus of the amygdala, corticosterone was suggested to activate a $\mathrm{G}_{\mathrm{s}}$-associated membrane receptor and to induce endocanna- binoid release, which eventually facilitates the consolidation of aversive memories (Campolongo et al, 2009). In the hippocampus, corticosterone can boost glutamate release and promote neuronal excitability through a nongenomic MR pathway (Karst et al, 2005). To the best of our knowledge, we are the first to provide functional evidence for the presence of membrane-bound GRs in the hippocampus. We show here that the acute application of DEX elicits a surprisingly rapid facilitation of GABAergic transmission in the hippocampus of control animals. The fast onset of the DEX effect shown in this study is substantially different from the time course of DEX effect described in a recent paper (Maggio and Segal, 2009). There, the authors reported that DEX caused an increase in the amplitude but not in the frequency of sIPSCs in the dorsal hippocampus of the rat. More importantly, Maggio and Segal (2009) described a slow onset and long-lasting response, detected 30-45 min after DEX application. They suggested that this slow effect was caused by the activation of genomic GR. In this paper, the fast DEX effect on sIPSCs, which act through the nongenomic mechanism, occurs within a narrow time window (about 5-10 min after DEX application, see Figure 1). There are at least three 
a1
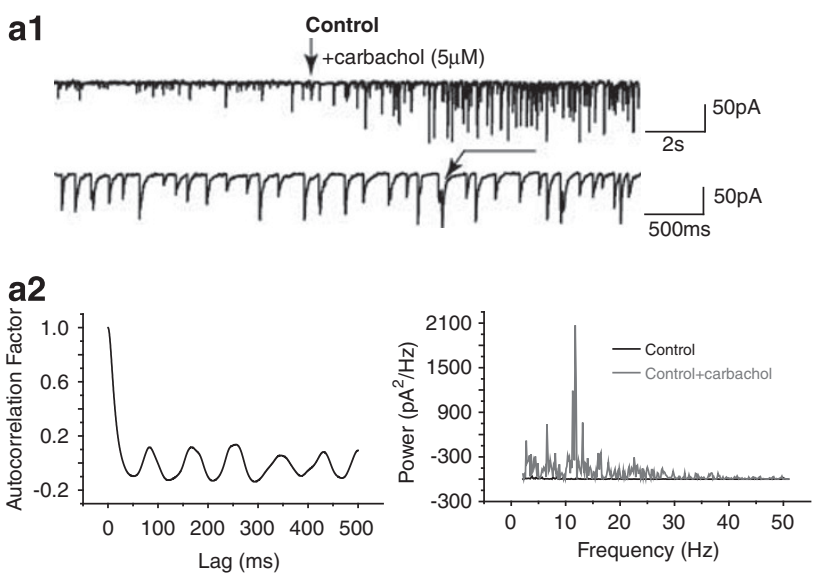

c1
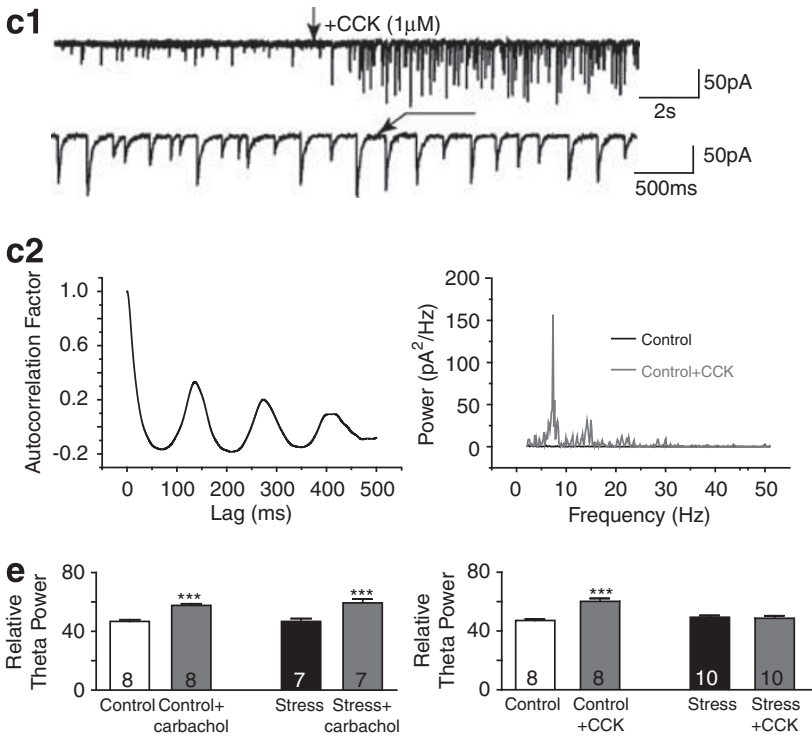

b1

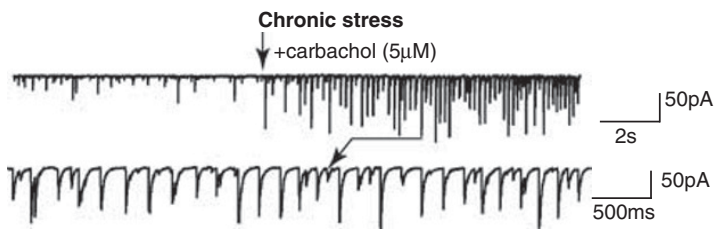

b2
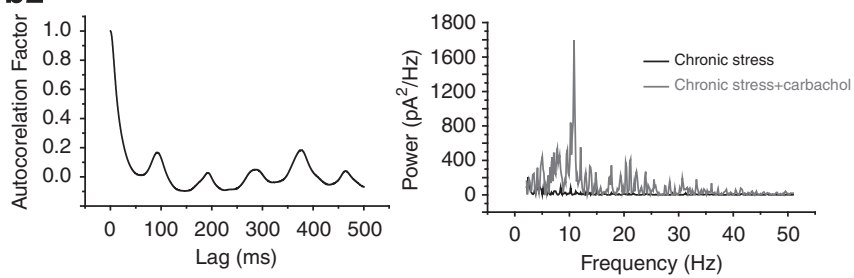

d1

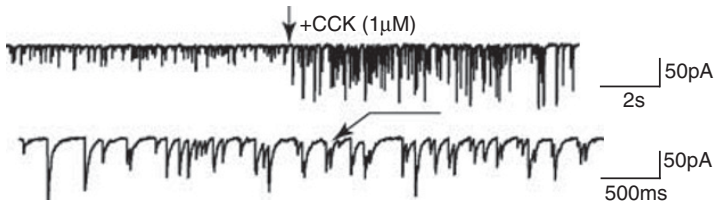

d2
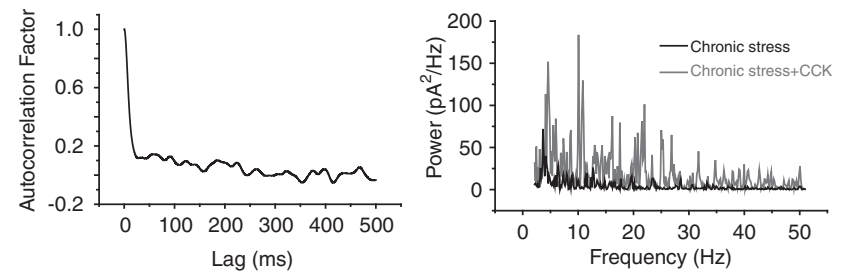

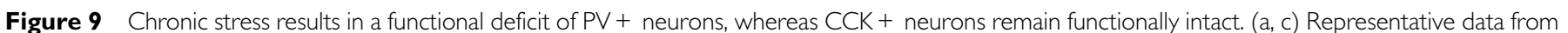

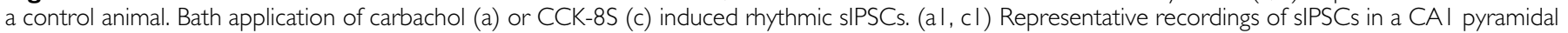

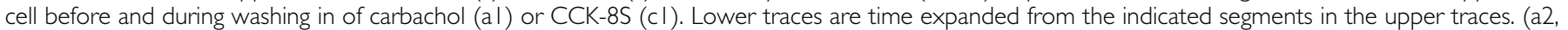

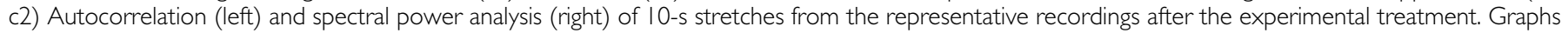

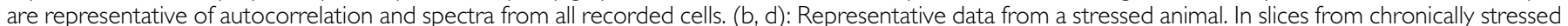

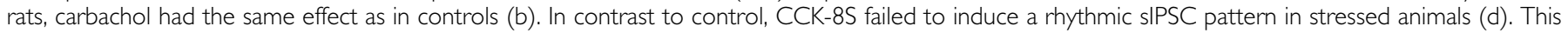

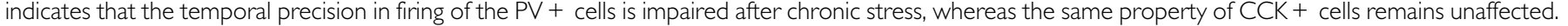

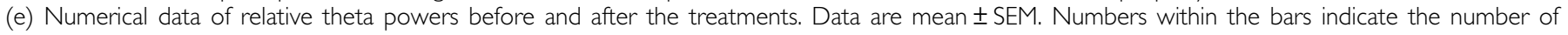
neurons tested for each animal group (seven animals per group) and experimental condition, with the same cells in all conditions; **** $<0.00$ I.

possibilities that can explain the discrepancy between the two studies. (i) In the study by Maggio and Segal (2009), the perfusion rate during the recordings was quite slow, which meant that the equilibrium could be reached only after $6 \mathrm{~min}$, whereas in our case the perfusion rate was much faster; thus the equilibrium could be reached already within $1 \mathrm{~min}$. The slow perfusion rate may confound the fast onset of DEX effect. (ii) The numerical values of sIPSC frequency and amplitude were calculated as the total number of events in $7 \mathrm{~min}$ of recording epochs (Maggio and Segal, 2009), which exceeds the narrow time window of the fast DEX effect shown in Figure 1. (iii) In the study by Maggio and Segal (2009), the sIPSCs were recorded using a CsClcontaining pipette solution. This may explain why the recorded sIPSCs were small in amplitude and sparse in frequency. Therefore, the fast-onset enhancement of sIPSCs in response to DEX may have been concealed.
We show here that the DEX effect is mediated by a membrane-bound receptor as the membrane-impermeable BSA-DEX retained the effect. In addition, activation of the nongenomic GRs induces downstream retrograde NO signaling, which eventually augments GABAergic transmission from presynaptic interneurons. The involvement of NO in modulating GABAergic transmission is further supported by studies showing that hippocampal GABAergic synapses possess the molecular machinery for retrograde $\mathrm{NO}$ signaling (Szabadits et al, 2007). Furthermore, NO signaling has been reported to facilitate action potentials in the peptidergic neurons of the posterior pituitary (Klyachko et al, 2001). It is tempting to speculate that NO signaling induced by nongenomic GR action in the hippocampus also modulates the opening of ion channels involved in certain phase(s) of an action potential; therefore, mIPSCs were not affected by DEX in our experiment. Taken together, our 
results suggest that the activation of nongenomic GRs, by evoking retrograde NO signaling, facilitates the firing activity of the hippocampal interneurons. Given the association between GABAergic inhibition and network oscillation, the cross talk between glucocorticoids and NO signaling may have a role in boosting oscillations to enhance cortical circuit performance in stressful situations.

Furthermore, we observed that the DEX-induced facilitation of GABA release was partially blocked by the $\mathrm{CCK}_{2}$ receptor antagonist LY225910. Moreover, the facilitating effect of the NO donor SNAP on sIPSCs was also significantly reduced in the presence of LY225910. The most likely interpretation for this is that DEX induces retrograde NO signaling, which activates the NO-sensitive guanylyl cyclase and thus leads to cGMP production. On the one hand, cGMP increases GABA transmission from both $\mathrm{PV}+$ and CCK + cells, and on the other, it induces CCK release from $\mathrm{CCK}+$ cells. However, it remains to be elucidated how cGMP is associated with CCK release. As activation of $\mathrm{CCK}_{2}$ receptors massively increases the activity of PV + cells (Földy et al, 2007), the endogenous CCK should also contribute to the facilitating effect of DEX on GABA release (Figure $3 \mathrm{~b}$ ). This interpretation is in line with recent data showing that stress increases cortical CCK release (Becker et al, 2008).

\section{Stress Increases GABAergic Transmission in the Hippocampus}

Here, we show for the first time that exposing animals to acute stress modulates the GABAergic input to CA1 pyramidal neurons. The effect of acute stress on the occurrence of sIPSCs was partially mimicked by DEX application to control slices. Both acute stress and DEX increased the frequency of sIPSCs to a similar extent, whereas the amplitude of sIPSC was affected only by DEX. In addition, in our ex vivo experimental condition, the DEX-induced facilitation of sIPSCs appeared to be relatively brief and started to dwindle already $\sim 10 \mathrm{~min}$ after initial exposure to the agonist, whereas the animals in the acute stress group were subjected to restraint for $30 \mathrm{~min}$. The similarity and diversity between the effect of DEX and acute stress are not surprising. On the one hand, corticosterone is the main stress hormone in rodents; on the other, a stress response involves the release of various other 'stress mediators' that act in concert along with corticosterone. In addition, together with the data by Maggio and Segal (2009), it is possible that an acute stress response collectively involves fast nongenomic GR action as elucidated in this study, as well as genomic GR actions that emerge after $\sim 30 \mathrm{~min}$ of stress exposure.

Furthermore, we provide the first electrophysiological evidence showing that the altered hippocampal GABAergic transmission sustains following a prolonged period of stress. Three weeks of daily restraint stress induced an elevation in $\mathrm{Ca}^{2+}$-dependent sIPSC frequency, and the membrane-permeable $\mathrm{Ca}^{2+}$ chelator EGTA-AM was able to compensate this stress effect. This is in line with previous findings that chronic stress and glucocorticoid treatments regulate hippocampal GAD expression, which presumably leads to enhanced GABA synthesis and hippocampal GABA receptor expression (Bowers et al, 1998; Stone et al, 2001).
One may argue that the increased interneuron activity observed in the stressed animals may be due to an increased excitatory drive to interneurons rather than an effect on interneurons themselves, as enhanced excitatory activity evoked by chronic stress has long been reported (Joëls et al, 2007; Joëls, 2008). However, this should not take credit for the observations under our experimental condition in which glutamatergic transmission was blocked by CNQX and APV in the bath to isolate inhibitory postsynaptic currents. In addition, the increased sIPSC frequency in chronically stressed animals may indicate an increased discharge of interneurons or an enhanced release probability at their terminals.

Importantly, the same concentration of DEX that elicited rapid facilitation of sIPSCs in the control hippocampal slices failed to induce any further increase in sIPSCs in the slices from the chronically stressed animals. This result can be explained by the following two possibilities: (i) The rapid nongenomic DEX-NO signaling pathway is continually activated during the entire period of chronic stress, which may contribute to the $\mathrm{Ca}^{2+}$-dependent enhancement of sIPSC frequency observed in the hippocampus of the stressed animals. It is likely that the ligand-binding domains of all the membrane-bound GRs are saturated because of a sustained increase in GC levels, and therefore the bath application of DEX failed to induce any further effect; or alternatively (ii), the DEX-NO signaling is destructed or desensitized after a certain period of stress exposure. However, during the time when this pathway is still active, sustained hyperactivity and enhanced $\mathrm{Ca}^{2+}$ influx into the interneurons might induce activity-dependent gene transcription through mechanisms such as epigenetics and progressively result in adaptive alterations in the expression levels or properties of proteins that are associated with GABA metabolism or transmission (eg, GAD) and/or ion channels that regulate the firing activity of the interneurons (eg, voltage-gated calcium channel). These secondary changes may underlie the observed chronic stress-induced elevation in hippocampal GABAergic transmission.

\section{Chronic Stress Results in a Functional Deficit of the PV + Neuronal Network, whereas CCK + Neurons Remain Largely Unchanged}

According to the theory of the functional dichotomy of PV + and CCK + neurons proposed by Freund and Katona (2003), one may assume that the functional integrity of $\mathrm{CCK}+$ neurons should be more affected in the chronically stressed animals. However, as discussed above, endogenous CCK is released in response to GC exposure. As CCK specifically stimulates $\mathrm{PV}+$ cells (Földy et al, 2007), it appears that during stress, stimulatory effects converge on the PV + cells in that they are affected by both NO and CCK. In this hub site, $\mathrm{PV}+$ cells appear to be in a more vulnerable position compared with CCK + cells. In support of this hypothesis, an earlier morphometric study suggested that PV + neurons are the ones vulnerable to stress (Czéh et al, 2005). Consistently, our present results show that after 3 weeks of restraint stress, the number of PV-immunoreactive cells was significantly reduced in all hippocampal subfields. Furthermore, the PV interneuron network of stressed animals exhibited a functional deficit in that it 

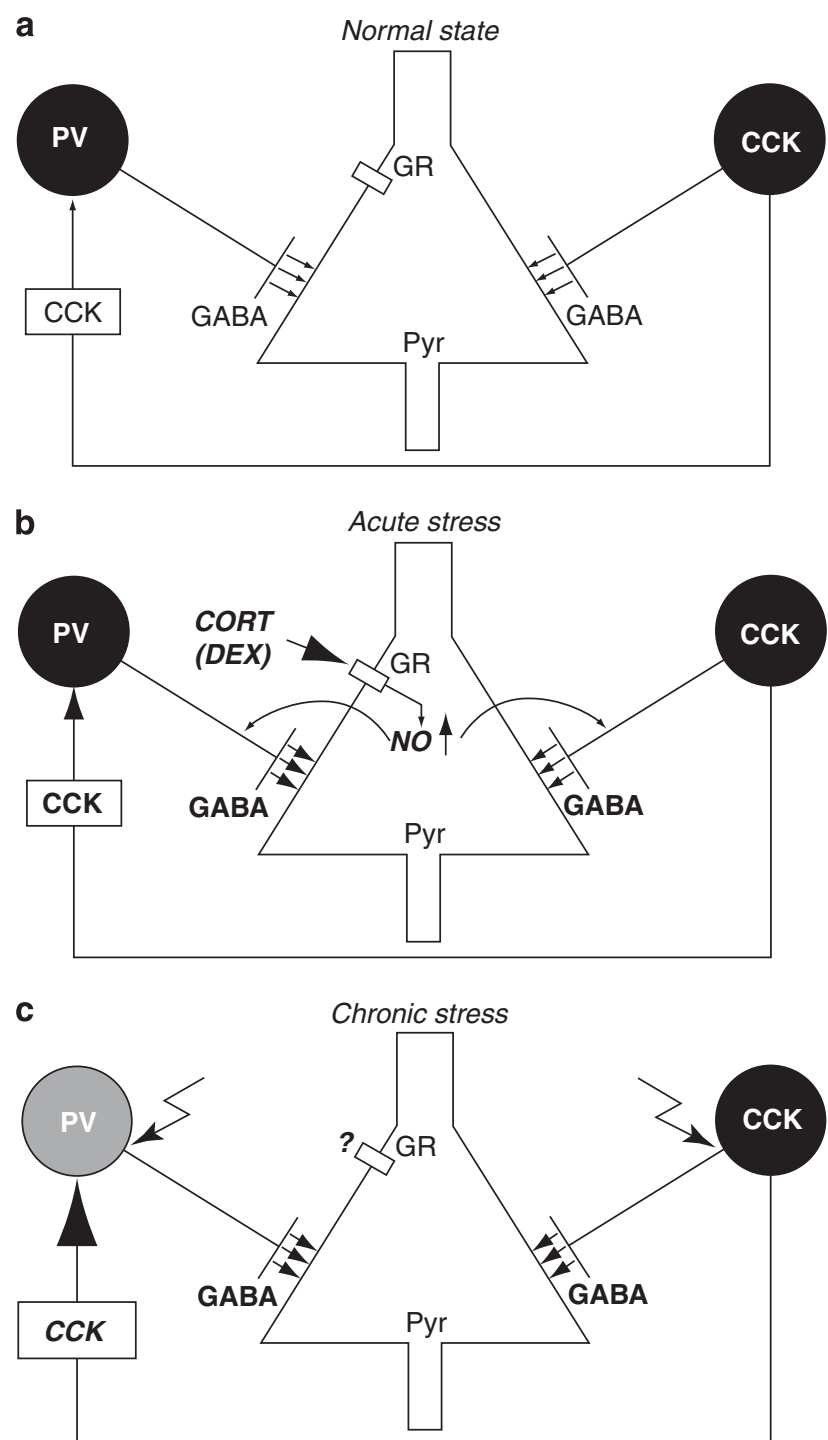

Figure 10 Theoretical summary of the present findings. (a) Under control conditions, PV + and CCK + cells provide two parallel, functionally distinct sources of perisomatic GABAergic inhibition to the pyramidal cells. In addition, CCK, presumably released from the CCK + cells, stimulates the $\mathrm{PV}+$ cells. (b) In response to acute stress, the glucocorticoid surge rapidly activates membrane-bound GRs on pyramidal cells, which results in NO release. Retrograde NO signaling stimulates GABA release from the GABAergic terminals. In addition, $\mathrm{NO}$ induces release of CCK, which in turn stimulates PV + cells. (c) During chronic stress, the above mechanisms are continuously or repetitively activated, which results in sustained elevation of $\mathrm{Ca}^{2+}$ influx and GABA release. In this situation, the PV + cells are in a more vulnerable position as they receive stimulatory effects also from the CCK + cells. The persistently elevated $\mathrm{Ca}^{2+}$ level in the PV + cells is detrimental to their cellular integrity, and their capacity to generate rhythmic oscillations is impaired. Thus, chronic stress causes functional and structural deficits in PV + cells. Secondary adaptive alterations of proteins associated with GABAergic transmission are probably induced to facilitate sustained hyperactivity of interneurons. CCK, cholecystokinin; GR, glucocorticoid receptor; NO, nitric oxide; PV, parvalbumin; pyr, pyramidal neuron.

failed to generate rhythmic sIPSCs in response to the CCK analog.

This functional deficit of the PV interneuron network may reflect a dysfunction of $\mathrm{CCK}_{2}$ receptors. At this point, we cannot rule out this possibility. However, the bath application of CCK induced a massive increase in sIPSCs in both the control and chronic stress groups. It was only the rhythmicity of the CCK-induced sIPSCs that was impaired by chronic stress. On the other hand, application of EGTAAM could normalize chronic stress-induced elevation in the hippocampal sIPSC frequency, suggesting that the stressstimulated GABA transmission comes along with excessive intracellular free $\mathrm{Ca}^{2+}$. As reported earlier, an elaborately regulated $\mathrm{Ca}^{2+}$ signaling contributes to the synchronous release of GABA quanta from hippocampal PV + neurons (Caillard et al, 2000; Hefft and Jonas, 2005). Therefore, we hypothesize that during chronic stress, the prolonged elevation of corticosterone results in sustained high activity of PV + cells, which in turn leads to increased $\mathrm{Ca}^{2+}$ influx that is beyond the buffering capacity of the calcium binding protein PV expressed in those neurons. As a consequence, the high presynaptic $\mathrm{Ca}^{2+}$ level boosts GABA release from the $\mathrm{PV}+$ cells and results in inaccurately timed response patterns of these neurons.

\section{Conclusions}

The summary of our findings and a theoretical explanation are presented in Figure 10. Our data show that short-term exposure to DEX increases activity of hippocampal interneurons through retrograde signaling of $\mathrm{NO}$ and $\mathrm{CCK}$ (downstream from NO) by activating membrane-bound GRs in the pyramidal neurons. We suggest that the outlined nongenomic action of DEX provides a potential pathogenic mechanism that underlies the chronic stress-induced effects reported in this study, although the cellular mechanisms of acute glucocorticoid action and the chronic stress effects are likely to differ. We hypothesize that the continual activation of nongenomic GRs contributes to the development of the chronic stress-induced $\mathrm{Ca}^{2+}$-dependent elevation in GABAergic transmission. Furthermore, as hippocampal PV interneurons are affected by both NO and CCK, their excessive excitation may eventually develop into a cellular and functional deficit. Considering the vital role of PV + cells in the regulation of network oscillations (Somogyi and Klausberger, 2005; Sohal et al, 2009), the stress-induced deficits of these cells may underlie the altered oscillation patterns that are implicated in cognitive impairments, which are common in patients with stress-related psychiatric illnesses.

\section{ACKNOWLEDGEMENTS}

We thank C Heckmann for excellent technical assistance. This work was supported by the DFG-Research Center for Molecular Physiology of the Brain (CMPB; GF and WZ). $\mathrm{WH}$ received a Georg-Christoph-Lichtenberg stipend from the Ministry of Lower Saxony, Germany.

\section{DISCLOSURE}

The authors declare that, except for income received from primary employer, no financial support or compensation has been received from any individual or corporate entity over the past 3 years for research or professional service and that there are no personal financial holdings that could be perceived as constituting a potential conflict of interest. 


\section{REFERENCES}

Becker C, Zeau B, Rivat C, Blugeot A, Hamon M, Benoliel JJ (2008). Repeated social defeat-induced depression-like behavioral and biological alterations in rats: involvement of cholecystokinin. Mol Psychiatry 13: 1079-1092.

Bowers G, Cullinan WE, Herman JP (1998). Region-specific regulation of glutamic acid decarboxylase (GAD) mRNA expression in central stress circuits. J Neurosci 18: 5938-5947.

Brambilla P, Perez J, Barale F, Schettini G, Soares JC (2003). GABAergic dysfunction in mood disorders. Mol Psychiatry 8: 721-737, 715.

Buzsáki G, Draguhn A (2004). Neuronal oscillations in cortical networks. Science 304: 1926-1929.

Caillard O, Moreno H, Schwaller B, Llano I, Celio MR, Marty A (2000). Role of the calcium-binding protein parvalbumin in short-term synaptic plasticity. Proc Natl Acad Sci USA 97: 13372-13377.

Campolongo P, Roozendaal B, Trezza V, Hauer D, Schelling G, McGaugh JL et al (2009). Endocannabinoids in the rat basolateral amygdala enhance memory consolidation: Involvement of the glucocorticoid system. Proc Natl Acad Sci USA 106: 4888-4893.

Czéh B, Simon M, van der Hart MG, Schmelting B, Hesselink MB, Fuchs E (2005). Chronic stress decreases the number of parvalbumin-immunoreactive interneurons in the hippocampus: prevention by treatment with a substance $\mathrm{P}$ receptor (NK1) antagonist. Neuropsychopharmacology 30: 67-79.

de Groote L, Linthorst AC (2007). Exposure to novelty and forced swimming evoke stressor-dependent changes in extracellular GABA in the rat hippocampus. Neuroscience 148: 794-805.

De Kloet ER, Vreugdenhil E, Oitzl MS, Joëls M (1998). Brain corticosteroid receptor balance in health and disease. Endocr Rev 19: 269-301.

Di S, Malcher-Lopes R, Marcheselli VL, Bazan NG, Tasker JG (2005). Rapid glucocorticoid-mediated endocannabinoid release and opposing regulation of glutamate and gamma-aminobutyric acid inputs to hypothalamic magnocellular neurons. Endocrinology 146: 4292-4301.

Di S, Maxson MM, Franco A, Tasker JG (2009). Glucocorticoids regulate glutamate and GABA synapse-specific retrograde transmission via divergent nongenomic signaling pathways. J Neurosci 29: 393-401.

Drevets WC, Price JL, Furey ML (2008). Brain structural and functional abnormalities in mood disorders: implications for neurocircuitry models of depression. Brain Struct Funct 213: 93-118.

Földy C, Lee SY, Szabadics J, Neu A, Soltesz I (2007). Cell typespecific gating of perisomatic inhibition by cholecystokinin. Nat Neurosci 10: 1128-1130.

Freund TF, Katona I (2003). Perisomatic Inhibition. Neuron 56: 33-42.

Haller J, Mikics E, Makara GB (2008). The effects of nongenomic glucocorticoid mechanisms on bodily functions and the central neural system. A critical evaluation of findings. Front Neuroendocrinol 29: 273-291.

Hasler G, van der Veen JW, Tumonis T, Meyers N, Shen J, Drevets WC (2007). Reduced prefrontal glutamate/glutamine and gamma-aminobutyric acid levels in major depression determined using proton magnetic resonance spectroscopy. Arch Gen Psychiatry 64: 193-200.

Hebb AL, Poulin JF, Roach SP, Zacharko RM, Drolet G (2005). Cholecystokinin and endogenous opioid peptides: interactive influence on pain, cognition, and emotion. Prog Neuropsychopharmacol Biol Psychiatry 29: 1225-1238.

Hefft S, Jonas P (2005). Asynchronous GABA release generates long-lasting inhibition at a hippocampal interneuron-principal neuron synapse. Nat Neurosci 8: 1319-1328.
Joëls M (2008). Functional actions of corticosteroids in the hippocampus. Eur J Pharmacol 583: 312-321. Review.

Joëls M, Karst H, Krugers HJ, Lucassen PJ (2007). Chronic stress: implications for neuronal morphology, function and neurogenesis. Front Neuroendocrinol 28: 72-96.

Karst H, Berger S, Turiault M, Tronche F, Schütz G, Joëls M (2005). Mineralocorticoid receptors are indispensable for nongenomic modulation of hippocampal glutamate transmission by corticosterone. Proc Natl Acad Sci USA 102: 19204-19207.

Karson MA, Whittington KC, Alger BE (2008). Cholecystokinin inhibits endocannabinoid-sensitive hippocampal IPSPs and stimulates others. Neuropharmacology 54: 117-128.

Klausberger T, Marton LF, O'Neill J, Huck JH, Dalezios Y, Fuentealba $\mathrm{P}$ et al (2005). Complementary roles of cholecystokinin- and parvalbumin-expressing GABAergic neurons in hippocampal network oscillations. J Neurosci 25: 9782-9793.

Klyachko VA, Ahern GP, Jackson MB (2001). cGMP-mediated facilitation in nerve terminals by enhancement of the spike afterhyperpolarization. Neuron 31: 1015-1025.

Krystal JH, Sanacora G, Blumberg H, Anand A, Charney DS, Marek $\mathrm{G}$ et al (2002). Glutamate and GABA systems as targets for novel antidepressant and mood-stabilizing treatments. Mol Psychiatry 7: S71-S80.

Lewis DA, Hashimoto T, Volk DW (2005). Cortical inhibitory neurons and schizophrenia. Nat Rev Neurosci 6: 312-324.

Lodge DJ, Behrens MM, Grace AA (2009). A loss of parvalbumincontaining interneurons is associated with diminished oscillatory activity in an animal model of schizophrenia. J Neurosci 29: 2344-2354.

Magariños AM, McEwen BS (1995). Stress-induced atrophy of apical dendrites of hippocampal CA3c neurons: involvement of glucocorticoid secretion and excitatory amino acid receptors. Neuroscience 69: 89-98.

Maggio N, Segal M (2009). Differential corticosteroid modulation of inhibitory synaptic currents in the dorsal and ventral hippocampus. J Neurosci 29: 2857-2866.

McEwen BS (2007). Physiology and neurobiology of stress and adaptation: central role of the brain. Physiol Rev 87: 873-904.

McEwen BS, De Kloet ER, Rostene W (1986). Adrenal steroid receptors and actions in the nervous system. Physiol Rev 66: 1121-1188.

McLaughlin KJ, Gomez JL, Baran SE, Conrad CD (2007). The effects of chronic stress on hippocampal morphology and function: an evaluation of chronic restraint paradigms. Brain Res 1161: 56-64.

Pittenger C, Duman RS (2008). Stress, depression, and neuroplasticity: a convergence of mechanisms. Neuropsychopharmacology 33: 88-109.

Sanacora G, Mason GF, Rothman DL, Behar KL, Hyder F, Petroff OA et al (1999). Reduced cortical gamma-aminobutyric acid levels in depressed patients determined by proton magnetic resonance spectroscopy. Arch Gen Psychiatry 56: 1043-1047.

Sohal VS, Zhang F, Yizhar O, Deisseroth K (2009). Parvalbumin neurons and gamma rhythms enhance cortical circuit performance. Nature 459: 698-702.

Somogyi P, Klausberger T (2005). Defined types of cortical interneurone structure space and spike timing in the hippocampus. J Physiol 562: 9-26.

Stone DJ, Walsh JP, Sebro R, Stevens R, Pantazopolous H, Benes FM (2001). Effects of pre- and postnatal corticosterone exposure on the rat hippocampal GABA system. Hippocampus 11: 492-507.

Szabadits E, Cserép C, Ludányi A, Katona I, Gracia-Llanes J, Freund TF et al (2007). Hippocampal GABAergic synapses possess the molecular machinery for retrograde nitric oxide signaling. J Neurosci 27: 8101-8111. 